

\title{
SCARRING OF QUASIMODES ON HYPERBOLIC MANIFOLDS
}

\author{
SURESH ESWARATHASAN AND LIOR SILBERMAN
}

\begin{abstract}
Let $N$ be a compact hyperbolic manifold, $M \subset N$ an embedded totally geodesic submanifold, and let $-\hbar^{2} \Delta_{N}$ be the semiclassical Laplace-Beltrami operator.

For any $\varepsilon>0$ we explicitly construct families of quasimodes of energy width at most $\varepsilon \frac{\hbar}{|\log \hbar|}$ which exhibit a "strong scar" on $M$ in that their microlocal lifts converge weakly to a probability measure which places positive weight on $S^{*} M\left(\hookrightarrow S^{*} N\right)$. An immediate corollary is that any invariant measure on $S^{*} N$ occurs in the ergodic decomposition of the semiclassical limit of certain quasimodes of width $\varepsilon \frac{\hbar}{|\log \hbar|}$.
\end{abstract}

\section{INTRODUCTION}

We consider a problem in the spectral asymptotics of the Laplace-Beltrami operator $\Delta_{N}$ on a compact Riemannian manifold $N$. Following the "semiclassical" convention we will index our eigenvalues and (approximate) eigenfunctions by a spectral parameter $\hbar$ tending to zero (the corresponding eigenvalue being $\lambda_{\hbar}=\hbar^{-2}$ ). Abusing notation we may have $\hbar$ tend to zero along a discrete sequence of values without making this explicit.

As discussed in greater detail below, many results on the concentration behaviour of exact eigenfunctions apply to certain approximate eigenfunctions as well and we address here the converse problem of constructing approximate eigenfunctions with prescribed concentration behavior. We start by specifying the relevant notion of "approximate eigenfunction", a relaxation of the eigenfunction equation $-\Delta_{N} \Psi_{\hbar}=\frac{E_{\hbar}}{\hbar^{2}} \Psi_{\hbar}$ :

Definition 1.1. Fix $C>0$ and a sequence of energies $\left\{E_{\hbar}\right\}_{\hbar}$ tending to $E_{0}>0$. A family of quasimodes of width $\frac{C \hbar}{|\log \hbar|}$ with central energies $E_{\hbar}$ is a sequence $\left\{\Psi_{\hbar}\right\}_{\hbar}$ of $L^{2}(N)$-normalized functions on $N$ such that

$$
\left\|\left(-\hbar^{2} \Delta_{N}-E_{\hbar}\right) \Psi_{\hbar}\right\|_{L^{2}(N)} \leq \frac{C \hbar}{|\log \hbar|} .
$$

If we prefer not to specify $C$ we will use the term "log-scale quasimodes". In the setting of our article, we will eventually set $E_{\hbar}=1+\mathcal{O}(h)$ for all $\hbar$. Finally, note the existence of such a non-zero quasimode as above shows that $-\hbar^{2} \Delta_{N}$ has an eigenvalue $E$ in the interval $\left[E_{\hbar}-\frac{C \hbar}{|\log \hbar|}, E_{\hbar}+\frac{C \hbar}{|\log \hbar|}\right]$.

We will study measure-theoretic concentration in the weak-* limit. Precisely, to a quasimode $\Psi_{\hbar}$ we associate the linear functional $\bar{\mu}_{\hbar}$ on $N$ given by $\bar{\mu}_{\hbar}(f)=\int_{N}\left|\Psi_{\hbar}\right|^{2} f d V$ for test functions $f$ on $N$, where $d V$ is the Riemannian volume form on $N$.

These measures have natural lifts to distributions $\mu_{\hbar}$ on the cotangent bundle $T^{*} N$ (commonly referred to as "microlocal lifts"); we review the construction later. A weak-* limit of these microlocal lifts is necessarily a probability measure on $T^{*} N$. These limits, to be denoted $\mu_{\mathrm{sc}}$ and called quantum limits or semiclassical measures, are the subject of this paper. Informally, they may be called weak-* limits of the quasimodes themselves. 
The reader is advised that these terms (quantum limit and semiclassical measure) are usually reserved for the case where the $\Psi_{\hbar}$ are exact eigenfunctions, but the more general use is appropriate here. We will be clear in each invocation of these terms.

Our main result is the following

Theorem (Cor. 1.10). Let $N$ be a compact hyperbolic manifold (that is, a compact Riemannian manifold of constant negative curvature) then there exists $C>0$ such that any probability measure $\mu$ on the unit cotangent bundle $S N$, which is invariant under the geodesic flow, arises as the quantum limit of quasimodes of width $\frac{C \hbar}{|\log \hbar|}$.

In the next part of the introduction we motivate our work by reviewing the quantum unique ergodicity problem and various results towards it. Knowledgeable readers may wish to skip to Section 1.3 where we discuss all our results.

1.1. The quantum unique ergodicity problem. The cotangent bundle $T^{*} N$ is naturally the phase space for a single particle moving on our manifold $N$. We fix a quantization scheme $\mathrm{Op}_{\hbar}$, assigning to each observable, which is a smooth function $a$ on $T^{*} N$ belonging to an appropriate symbol class, an operator $\mathrm{Op}_{\hbar}(a): L^{2}(N) \rightarrow L^{2}(N)$. Fix a positive observable $H$, called the Hamiltonian, and suppose that for a sequence of values of $\hbar$ tending to zero we have chosen a corresponding sequence of normalized eigenfunctions $\Psi_{\hbar} \in L^{2}(N)$ where

$$
\mathrm{Op}_{\hbar}(H) \Psi_{\hbar}=E_{\hbar} \Psi_{\hbar} .
$$

Let us suppose that $E_{\hbar}=E_{0}+\mathcal{O}(\hbar)$ for some fixed $E_{0}>0$. To each $\Psi_{\hbar}$, we associate its Wigner measure which is the distribution $\mu_{\hbar}$ on $T^{*} N$ given by

$$
\mu_{\hbar}(a)=\left\langle\Psi_{\hbar}, \mathrm{Op}_{\hbar}(a) \Psi_{\hbar}\right\rangle .
$$

A major problem in spectral asymptotics is to study the concentration behavior of the eigenfunctions $\Psi_{\hbar}$ - the basic expectation is that the more chaotic the classical dynamics induced by $H$, the more uniformly distributed the eigenfunctions $\Psi_{\hbar}$ are as $\hbar \rightarrow 0$. One tool for studying this problem is the examination of the weak-* limit points of the $\mu_{\hbar}$, and it is these objects that we call "semiclassical measures" or "quantum limits" $\mu_{\text {sc }}$. Their study was initiated in the works of Schnirel'man, Zelditch, and Colin de Verdière [28, 30, 10], which we describe in more detail shortly.

Let us review some aspects of quantization schemes. First, since two different quantization schemes differ by terms of order $\mathcal{O}(\hbar)$, it follows that the measure $\mu_{\text {sc }}$ depends on the sequence $\Psi_{\hbar}$ but not on the scheme itself. Second, the existence of a positive quantization scheme (the so-called Friedrich symmetrization) shows that any limit must be a positive measure. Using for $a$ the constant function 1 - for which $\mathrm{Op}_{\hbar}(a)$ is the identity operator - shows that our limit $\mu_{\mathrm{sc}}$ is a probability measure. Standard techniques also show that the measure $\mu_{\mathrm{sc}}$ must be supported on the energy surface $\left\{H(x, \xi)=E_{0}\right\} \subset T^{*} N$. Third, Egorov's Theorem relates the Hamiltonian flow induced by $H$ to the action of the Schrödinger propagator $U(t)=$ $\exp \left(-\frac{i t}{\hbar} \mathrm{Op}_{\hbar}(H)\right)$. Since replacing $\Psi_{\hbar}$ with its time-evolved state $U(t) \Psi_{\hbar}=\exp \left(-\frac{i t E_{\hbar}}{\hbar}\right) \Psi_{\hbar}$ has no effect on $\mu_{\hbar}$ one can show that any limit $\mu_{\mathrm{sc}}$ must be invariant under the Hamiltonian flow generated by $H$. For a more general discussion of these properties, see the book [32] by Zworski. 
Problem 1.3 (Quantum Unique Ergodicity ("QUE")). Classify, amongst the flow-invariant measures supported on level sets of $H$, those which are weak-* limits of sequences of Wigner measures of eigenfunctions.

The case of a free particle moving on a compact Riemmanian manifold where the classical dynamics is geodesic flow corresponds to the Hamiltonian $H(x, \xi)=\|\xi\|_{g}^{2}$; here, we identify the tangent and cotangent bundles using the metric $g$. Its semiclassical quantization $\mathrm{Op}_{\hbar}(H)$ is $^{1}-\hbar^{2} \Delta_{g}$, where $\Delta_{g}$ is the Laplace-Beltrami operator for the metric $g$. The eigenfunction equation (1.2) is then equivalent to the familar spectral problem $-\Delta \Psi=\lambda \Psi$ where we take $\hbar=\sqrt{\frac{1}{\lambda}}$ and $E_{\hbar}=1$. For non-zero $E_{0}$, the energy surfaces $\left\{H=E_{0}\right\}$ are then all just dilations of the unit tangent bundle $S N$, which we call our energy surface. The metric on $N$ naturally gives rise to a geodesic-flow invariant measure, the Liouville measure $\mu_{L, N}$.

We briefly revert to indexing the eigenfunctions of $\Delta_{N}$ in accordance with their set of non-decreasing eigenvalues so that we may state the following classical theorems:

THEOREM 1.4. Let $N$ be a compact Riemannian manifold, let $\left\{\Psi_{n}\right\}_{n=0}^{\infty} \subset L^{2}(N)$ be an orthonormal basis of eigenfunctions of $\Delta_{N}$ with corresponding eigenvalues $\left\{\lambda_{n}\right\}_{n=0}^{\infty}$. Write $\mu_{n}$ for the Wigner measure associated to the eigenfunction $\Psi_{n}$. We have that

(1) (Convergence on average/Generalized Weyl law) As distributions on $T^{*} N$,

$$
\frac{1}{C_{N} T^{n}} \sum_{n<T} \mu_{n} \underset{T \rightarrow \infty}{\stackrel{w k^{*}}{\longrightarrow}} \mu_{L, N},
$$

for some $C_{N}>0$ a constant.

(2) ("Quantum ergodicity") If the Liouville measure $\mu_{L, N}$ is ergodic with respect to the geodesic flow, then there exists a subsequence $\left\{\Psi_{n_{k}}\right\}_{k=0}^{\infty}$ of density one along which the Wigner measures converge to $\mu_{L, N}$. Stated otherwise, "almost all" eigenfunctions asymptotically equidistribute on $S N$.

A major case where the Liouville measure is ergodic is that of negatively curved manifolds where we further have

Conjecture 1.5 (Quantum Unique Ergodicity; Rudnick-Sarnak [25]). For $N$ compact of negative sectional curvature, we have $\mu_{n} \underset{n \rightarrow \infty}{\stackrel{w k^{*}}{\longrightarrow}} \mu_{L, N}$. In other words, the Liouville measure is the unique quantum limit.

This conjecture predicts a form of uniform distribution of the eigenfunctions. The opposite behavior, meaning an enhancement of some eigenfunctions along closed geodesics, was observed numerically by Heller [19] in the case of plane billiards and named "scarring". Perhaps the strongest form of this phenomenon, a "strong scar" along an invariant measure, is the situation where a semiclassical measure has this particular measure along a closed geodesic as an atom in its ergodic decomposition.

Some ergodic Euclidean billiards were shown to be quantum ergodic in the sense of Theorem 1.4 by Gérard-Leichtnam [17] and Zelditch-Zworski [31]. The question of whether the numerically observed scarring persists in the semiclassical limit for ergodic billiards was

\footnotetext{
${ }^{1}$ Formally, this holds up to an operator of order $\mathcal{O}_{L^{2}}(h)$ - perhaps it is better to use the converse formulation that the principal symbol of $-h^{2} \Delta_{g}$ is $|\xi|_{g}^{2}$.
} 
mostly settled by Hassell, who showed in [18] that for the one-parameter family of billiards known as the Bunimovich stadium, almost every member has a sequence of eigenfunctions whose quantum limit puts positive mass on the set of "bouncing ball" trajectories, a oneparameter family of closed geodesics. In negative curvature, closed orbits are unstable so such families of trajectories cannot exist. However, it is still an open question as to whether semiclassical measures on the stadium contain scars, particularly on the single closed orbit with endpoints on the circular wings.

Positive results toward the Rudnick-Sarnak Conjecture were obtained by Lindenstrauss [23] in the case of hyperbolic surfaces with the surface and the eigenfunctions enjoying additional arithmetic symmetries; we note that these arguments were recently simplified by Brooks-Lindenstrauss in [9]. To the knowledge of the authors, all positive results on general manifolds have depended on the breakthrough of Anantharaman [2]. She showed that for manifolds with geodesic flows having the Anosov property, quantum limits must have positive entropy with respect to the action of the geodesic flow, which in particular rules out the possibility of very singular measures being quantum limits. For example, a measure supported only a union of closed geodesics cannot occur as a semiclassical measure. Follow-up works with generalizations and improvements include [6, 4, 7, 3, 24].

The present article supports this line of work, specifically in studying to which extent these positive-entropy results are sharp.

1.2. Quasimodes. Many of the positive-entropy results discussed in the previous section continue to hold when one weakens the hypothesis that the $\boldsymbol{\Psi}_{\hbar}$ are exact eigenfuctions. In fact, the hypothesis that they are log-scale quasimodes in the sense of Definition 1.1 suffices. $^{2}$ For these we have that:

(1) Every quantum limit of a sequence of log-scale quasimodes is a probability measure supported on the energy surface $S N$.

(2) This measure is invariant under the geodesic flow.

(3) On a manifold of negative sectional curvature, any weak-* limit of log-scale quasimodes with $C>0$ small enough has positive entropy.

This version of Anantharaman's result is quantitative, in that the entropy bound depends on the spectral width parameter $C$. Conversely one may ask what is the smallest possible entropy of a quantum limit of a sequence of quasimodes with such a width, or more generally seek to classify those limits.

Problem 1.6 (QUE for quasimodes). Classify the weak-* limits of Wigner measures associated to log-scale quasimodes.

As we investigate this problem, we reserve the notation $\boldsymbol{\Psi}_{\hbar}$ for a sequence of quasidmoes for the rest of this paper. The first result concerning limits of quasimodes was obtained by Brooks:

THEOREM 1.7 ([8]). Let $N$ be a compact hyperbolic surface and $S \gamma \subset S N$ be the unit tangent bundle to a given closed geodesic. Then for any $\varepsilon>0$, there exists $\delta(\varepsilon)>0$ and a sequence of quasimodes of width $\frac{\varepsilon \hbar}{|\log \hbar|}$ and central energies $E_{\hbar}$ whose semiclassical measure $\mu_{s c}$ satisfies $\mu_{s c}(\{S \gamma\}) \geq \delta(\varepsilon)$.

\footnotetext{
${ }^{2}$ The constant $C$ may vary from one sequence to another.
} 
Brooks's construction is analogous to one of Faure-Nonnenmacher-De Bièvre [16] in the toy model known as the quantum cat map. However, while the argument in [16] is based on a coherent state decomposition, this particular result depends on the periodic boundary conditions on the hyperbolic surface and on the connection between eigenfunctions of the Laplace operator and the representation theory of $\mathrm{SL}_{2}(\mathbb{R})$.

Using microlocal techniques instead, Nonnenmacher and the first named author obtained a result for general Hamiltonians $H$ on a compact surface:

THEOREM 1.8 ([15]). Let $(N, g)$ be a compact Riemannian surface and $\gamma$ be a hyperbolic orbit of the Hamiltonian flow on an energy surface $H^{-1}\left(E_{0}\right)$ for some regular energy $E_{0}>$ 0 . Then for any $\varepsilon>0$ there is a sequence of quasimodes $\left\{\boldsymbol{\Psi}_{\hbar}\right\}_{\hbar}$ of width at most $\frac{\hbar}{|\log \hbar|}$ and central energies $E_{\hbar}=E_{0}+\mathcal{O}(\hbar /|\log \hbar|)$ of the quantum Hamiltonian $\mathrm{Op}_{\hbar}(H)$ whose semiclassical measure $\mu_{s c}$ has the property that $\mu_{s c}(\{S \gamma\}) \geq \frac{\varepsilon}{\pi \lambda_{\gamma}} \frac{2}{3 \sqrt{3}}+\mathcal{O}\left(\left(\varepsilon / \lambda_{\gamma}\right)^{2}\right)$. Here $\lambda_{\gamma}$ is the expansion rate along the unstable direction of the orbit $\gamma$.

1.3. Results of this paper. We seek to extend the above results to higher-dimensional manifolds (but only for the semiclassical Laplace-Beltrami operator), replacing the periodic geodesic $\gamma$ with a totally geodesic submanifold $M$, and achieve this goal when $N$ is a hyperbolic manifold. However, our techniques are mostly microlocal and there is reason to hope that they will apply in more general geometric settings.

The result is best thought of as a "transfer" principle: given a sequence of log-scale quasimodes on $M$ with associated quantum limit $\mu_{s c}$, we extend them transverally using the hyperbolic dynamics tranverse to $M$ to log-scale quasimodes on $N$ which still concentrate on $S M$ in the same manner as the original sequence, or at least give it positive mass. To obtain specifically the Liouville measure $\mu_{L, M}$ on $S M$, we use the equidistributed sequence provided by the Quantum Ergodicity Theorem. Our main result is the following:

THEOREM 1.9. Let $N$ be a complete hyperbolic manifold, let $M \subset N$ be an embedded compact totally geodesic submanifold, and pick a central energy $E_{0}>0$.

(1) Suppose we are given a sequence of central energies energies $E_{\hbar}=E_{0}+\mathcal{O}(\hbar)$ and some $\delta>0$. Select a width constant which satisfies $\pi \tilde{\lambda}_{E_{0}}(1+\delta) \geq C:=C_{M} \geq \pi \tilde{\lambda}_{E_{0}}$ where $\tilde{\lambda}_{E_{0}}=2 \sqrt{E_{0}}$ is the expansion rate in the unstable directions transverse to $M$. Then there exists a sequence of quasimodes $\left\{\boldsymbol{\Psi}_{\hbar}\right\}_{\hbar}$ of $N$ of width $\frac{C \hbar}{|\log \hbar|}$ for $\hbar \leq \hbar_{0}(M, \delta)$ and whose quantum limit is the Liouville measure on $S M$.

(2) Suppose in addition that $N$ is compact. Then for any $\varepsilon>0$ there exists a sequence of quasimodes with central energies converging to $E_{0}$ and of width $\frac{\varepsilon \hbar}{|\log \hbar|}$ whose quantum limit has $\mu_{L, M}$ as an ergodic component of mass at least $\eta=\eta\left(C_{M}, \varepsilon\right)>0$.

(3) In both cases one may replace $\mu_{L, M}$ with any semiclassical measure $\mu_{s c}$ on $S M$ arising from log-scale quasimodes on $M$ but at the cost of widening the quasimodes to the sum of the widths given in (1) (or (2)) and that of the quasimodes on $M$.

The bulk of the paper is devoted to establishing (1). The reference [15, Sec. 6] shows how to deduce (2) from (1) using spectral projection; see Lemma 7.7 below. As detailed in Lemma 4.5, the same arguments automatically establish (3) as well. Unlike the previous results for surfaces, our methods are not restricted by the dimension of $M$ or by the codimension of $N$ in $M$. 
We expect that the natural higher-dimensional generalization of Theorem 1.8 holds, with the only assumption that the dynamics transverse to $\mathrm{M}$ is hyperbolic; for more on this point see Remark 1.12 below. We also find that under the hypotheses of $\mathrm{N}$ compact and negatively curved it suffices to directly show concentration on closed geodesics (exactly the totally geodesic 1-dimensional submanifolds) in a strong sense: once concentration on arbitrary closed geodesics is achieved (with sufficient uniformity in the parameters), one can go beyond log-scale semiclassical measures supported on totally geodesic submanifolds and realize every invariant measure whatsoever as a log-scale semiclassical measure:

Corollary 1.10. Let $N$ be a compact hyperbolic manifold and let $\mu$ be a probability measure on $S N$ invariant by the geodesic flow. Then there is a sequence of log-scale quasimodes whose associated semiclassical measure is $\mu$. Furthermore, given $\varepsilon>0$ there is $\eta=\eta(\varepsilon)>0$ and a sequence of quasimodes on $N$ of width $\frac{\varepsilon \hbar}{|\log \hbar|}$ whose quantum limit $\mu$ carries weight at least $\eta(\varepsilon)$ on the component $\mu$.

Proof. It was shown by Sigmund [26] that there is a sequence $\left\{\gamma_{k}\right\}_{k=1}^{\infty}$ of periodic geodesics whose natural measures $\delta_{k}=\delta_{S \gamma_{k}}$ converge in the weak-* topology to $\mu$. For each $k$ let $\left\{\psi_{k, n}\right\}_{n=1}^{\infty}$ be a sequence of log-scale quasimodes on $N$ (all sequences of the same spectral width) whose weak-* limit is $\delta_{S \gamma_{k}}$ guaranteed by Theorem 1.9 (1). By the separability of the space $C(S N)$ dual to the space of measures, a diagonal argument - using the uniformity in the constants from Theorem $1.9(1)$ - gives a subsequence $\left\{\psi_{k_{i}, n_{i}}\right\}_{i=1}^{\infty}$ which is a sequence of log-scale quasimodes and which converges to the desired limit $\mu$. The spectral projection argument can again be used to shorten the spectral width to $\frac{\varepsilon \hbar}{|\log \hbar|}$ while keeping at least weight $\eta(\varepsilon)$ on the component $\mu$.

To the knowledge of the authors, this is the first result in the mathematical quantum chaos literature for Laplace-Beltrami operators which demonstrates that $o(\hbar)$ quasimodes (which yield invariant semiclassical measures, log-scale or not) can develop scars on fractal subsets. However, it is worth noting that in the work of Anantharaman-Nonnenmacher on the Walsh-quantized baker's map, there exists a sequence of eigenfunctions coverging to a fractal semiclassical measure; see [5]. As in the work of Brooks, our bounds explore the extent to which the mentioned entropy bounds on quasimodes are sharp.

It is important to add that the construction of quasimodes which localize along closed geodesics, or more generally along smooth invariant submanifolds, has a long and rich history. For a brief exposition of this history, see the introduction of [15] and the references therein.

1.4. Outline of the proof and further remarks. The main idea of our proof is to combine the long-time evolution idea which first originated in the work of Vergini-Schneider [29] and the Fermi-normal coordinate/quantum Birkhoff normal idea of Colin de Verdière-Parisse [11].

Let $N$ be a hyperbolic manifold and $M \subset N$ a compact totally geodesic submanifold. Then for $\varepsilon_{1}>0$ small enough, the universal cover of the $\varepsilon_{1}$-neighbourhood $N_{\varepsilon_{1}}(M) \subset N$ (thought of as a subset of the universal cover $\tilde{N}$ of $N$ ) is exactly the $\varepsilon_{1}$-neighbourhood $N_{\varepsilon_{1}}(\tilde{M})$. In particular, we have a well-defined nearest-neighbor projection $\pi: N_{\varepsilon_{1}}(M) \rightarrow M$ and a coordinate system $z \mapsto(\pi(z), x)$ identifying $N_{\varepsilon_{1}}(M)$ with a product $M \times B$ where $B$ is a Euclidean $\varepsilon_{1}$-ball of dimension $r=\operatorname{dim} N-\operatorname{dim} M$. In this system the metric takes the 
form of a warped product and we can separate the variables to get:

$$
\Delta_{N}=\frac{1}{1+|x|^{2}} \Delta_{M}+\Delta_{x}+\left(x \cdot \frac{\partial}{\partial x}\right)^{2}+(n-1)\left(x \cdot \frac{\partial}{\partial x}\right) .
$$

Here $\Delta_{M}$ is the Laplace-Beltrami operator on the hyperbolic manifold $M, \Delta_{x}$ is the Euclidean Laplace operator in the tranverse variable $x$ and $x \cdot \frac{\partial}{\partial x}=\sum_{i=1}^{r} x_{i} \frac{\partial}{\partial x_{i}}$ is the smooth radial differentiation operator written in Cartesian coordinates. The calculation is reproduced in Section 2.2 and crucially depends on the precise form and special symmetries of the hyperbolic metric.

For our desired log-scale quasimode we use the ansatz

$$
\boldsymbol{\Psi}_{\hbar}(z)=\psi_{\hbar}(x) \varphi_{\hbar}(\pi(z))
$$

where $\varphi_{\hbar}$ is an eigenfunction on $M$ of energy $E_{0}$, therefore making $\varphi_{\hbar}$ stationary for the Schrödinger propagator associated to $\Delta_{M}$. $\boldsymbol{\Psi}_{\hbar}$ will concentrate on $M$ exactly when $\psi_{\hbar}$ will concentrate on the origin of the $x$-plane. This is similar to the construction in [15] where the $\psi_{\hbar}$ were particular localized wave packets on $M$ of width $\frac{C_{\gamma} \hbar}{|\log \hbar|}$ so that their evolution (especially self-interference) was non-trivial and needed to be dealt with using a particular normal form due to Sjöstrand [27]; we will discuss this in greater detail shortly. The use of exact eigenfunctions on $M$, at least in the coordinates given by a Birkhoff normal form which introduce non-trivial phase shift factors, was seen in [15] where $M=S^{1}$, which is the simplest example of a closed totally geodesic submanifold.

Plugging $\boldsymbol{\Psi}_{\hbar}$ into the Schrödinger equation

$$
-\hbar^{2} \Delta_{N} \boldsymbol{\Psi}_{\hbar}=\left(E_{0}+f(\hbar)\right) \boldsymbol{\Psi}_{\hbar}
$$

gives that $\psi_{\hbar}$ must be a quasimode of central energy 0 for a second Schrödinger operator of the form

$$
-\hbar^{2} \Delta_{x}-\hbar^{2}\left(x \cdot \frac{\partial}{\partial x}\right)^{2}-(n-1) \hbar^{2}\left(x \cdot \frac{\partial}{\partial x}\right)-\frac{E|x|^{2}}{1+|x|^{2}}-f(\hbar)
$$

where $f(\hbar)=\mathcal{O}(\hbar)$. The term $(n-1) \hbar^{2}\left(x \cdot \frac{\partial}{\partial x}\right)$ is lower order in $\hbar$ and hence negligible in specific sense. Ignoring for the moment the second order term $\hbar^{2}\left(x \cdot \frac{\partial}{\partial x}\right)^{2}$ and using the approximation $\frac{|x|^{2}}{1+|x|^{2}} \approx|x|^{2}$ (appropriate since our operator will be applied to wave packets which are concentrated near $x=0$ ) we have approximately the operator

$$
-\hbar^{2} \Delta_{x}-E_{0}|x|^{2}
$$

commonly referred to as the inverted harmonic oscillator. This Hamiltonian is well-known to have log-scale quasimodes concentrating at the origin, which is a hyperbolic fixed point of the associated classical dynamics [12].

To realize this intuition rigorously we employ a Quantum Birkhoff Normal Form due to Iantchenko [21]. This transformation, given by a semiclassical Fourier integral operator, approximates our Schrödinger operator near its hyperbolic fixed point by an inverted Harmonic oscillator together with controlled higher-order corrections. This approach is not a novelty, but both the variation of $x \in \mathbb{R}^{r}$ rather than $\mathbb{R}$ and our desire for uniformity sufficient for the argument of Corollary 1.10 require working through the arguments with some care. 
Remark 1.12. While we generally rely on the constant curvature hypothesis in various ways, there is reason to hope that our result could be extended to the case where $N$ is a general manifold and $M \subset N$ is an invariant hyperbolic submanifold, that is a totally geodesic submanifold for which the dynamics along $M$ uniformly expand and contract the directions transverse to $M$. There would be significant difficulties in realizing such an argument, however.

We contemplate an argument of roughly the same shape: separating variables along and transverse to $M$ the Laplace operator should split approximately into parallel and transverse parts, and using the same ansatz as before (the product of an eigenfunction on $M$ and a transverse wavepacket) the parallel part should act as a potential term in a transverse Hamiltonian acting on the transverse wavepacket alone. Now using a Quantum Birkhoff Normal Form should conjugate the transverse Hamiltonian to a quantum harmonic oscillator with controlled lower-order terms allowing the construction of transverse quasimodes.

The last part of the argument is on the firmest ground: while as written our arguments in the transverse direction in terms of the Quantum Birkhoff Normal Form rely on the equality of all Lyapunov exponents, they primarily rely on the hyperbolic fixed point and ought to hold generally.

However, the two preliminary components of the argument would require significant further work. First, we used on an exact separation of variables that crucially depended on $N$ being a locally symmetric space. In the variable curvature setting this separation would be more complicated and certainly less explicit. The transverse operator and the "potential terms" arising from the eigenfunction on $M$ would no longer be uniform along $M$ (though their leading order contributions should not be too bad).

Second, the less explicit and non-uniform nature of the putative transverse operator would make constructing the Birkhoff Normal Form more difficult. This non-uniformity indeed cuts against the product ansatz above, but we note that for surfaces of variable curvature (the precessor work [15] quoted in Theorem 1.8 above) an appropriate quantum normal form was found.

Remark 1.13. While we mananged to construct log-scale quasimodes concentrating on general invariant measures, including those supported on fractals, it would be interesting to construct them directly without appealing to Sigmund's Theorem. This would allow us to better explore the quantitative relation between the spectral width of the quasimodes and the possible entropies of the associated quantum limits: with the present method even to obtain concentration on measures of relatively large entropy we require the relatively large spectral width necessary to obtain concentration on closed geodesics.

Remark 1.14. We note that in spite of our highly symmetric setting of constant negative curvature, it is non-trivial to obtain entropy lower bounds for measures associated to our sequence of quasimodes of width $\frac{\varepsilon \hbar}{|\log \hbar|}$ where we can non-trivially and explicitly track the $\varepsilon$ dependence in the lower bound. While it is natural to believe that letting $\varepsilon$ tend to 0 will give us a form of Anantharaman's explicit lower bound, this would require transposing our construction into that of [2] (or possibly [6]) and doing a precise analysis of various constants appearing in the estimates.

We also remark that the results in [6],[4] work only for sub-logscale quasimodes but achieve better entropy bounds than those in the earlier work [2], yet the latter work contains results 
for quasimodes of width $\frac{\varepsilon \hbar}{|\log \hbar|}$ with slighty less sharp bounds. Essentially, we seek to make more explicit the results in [2] (or rather, extend the results in [6] to the case of logscale quasimodes) in the case of our particular logscale quasimodes. This is an ongoing project of the first named author and Stéphane Nonnenmacher.

Acknowledgements. The authors would like to thank Alex Gorodnik, Joanna Karczmarek, Brian Marcus, Jens Marklof and Roman Schubert for helpful discussions and the School of Mathematics at the University of Bristol for their hospitality during an important stage in the development of this paper. The second named author would also like to thank Peter Sarnak for originally suggesting this problem. During the writing of this article, the first named author was supported by a 10th Anniversary Grant from the Heilbronn Institute of Mathematical Research and the second was supported by an NSERC Discovery Grant.

\section{Coordinates And Decompositions}

In this section we discuss the hyperbolic geometry from the symmetric space point of view and provide an explicit Fermi normal coordinate system near our selected totally geodesic submanifold $M$, which will allow us to re-write the operator $\hbar^{2} \Delta_{N}$ with respect to a warpedproduct structure. This is the higher dimensional analogue of the idea in Section 6 of the work of Colin de Verdière-Parisse [11].

Remark 2.1. Identifying the tangent and cotangent bundles using the Riemannian metric, we may use the notations $T N$ and $T^{*} N$ (along with those for natural sub-bundles) interchangeably.

2.1. A non-quantitative collar Lemma. Our setup is as follows:

- Let $G$ be a semisimple Lie group with Iwasawa decomposition $G=N A K, H<G$ be a semisimple closed subgroup containing $A$ such that $K_{H}=K \cap H$ is a maximal compact subgroup of $H$.

- Let $S_{H}=H / K_{H}$ and $S=G / K$ be the corresponding symmetric spaces; note that $S_{H}$ embeds in $S$ as a totally geodesic submanifold.

- Let $\Gamma<G$ be a discrete subgroup such that $\Gamma_{H}=\Gamma \cap H$ is a uniform lattice in $H$.

LemMa 2.2. After passing to a finite-index subgroup we may assume $\Gamma_{H} \backslash(\Gamma \cap H K)=\{1\}$.

Proof. Let $\mathcal{F}_{H}$ be a fundamental domain for $\Gamma_{H} \backslash H$. Then the finite set $\Gamma \cap \mathcal{F}_{H} K$ is a set of representatives for the quotient. Since $\Gamma$ is residually finite there is a subgroup $\Gamma^{1}$ not containing these elements (except for the identity), such that $\Gamma^{1} \cap H K=\Gamma^{1} \cap H=\Gamma_{H}^{1}$.

Assumption 2.3. For the rest of this article, we let $M=\Gamma_{H} \backslash H / K_{H}$ be embedded in $N=$ $\Gamma \backslash G / K$.

Now, let $\pi: S \rightarrow S_{H}$ be the projection onto a convex subset, and write $N_{\varepsilon}\left(S_{H}\right)$ to be the $\varepsilon$-neighborhood of $S_{H}$ in $S$.

LEMMA 2.4. There exists an $\varepsilon$ such that if $\gamma \in \Gamma$ has $\gamma N_{\varepsilon}\left(S_{H}\right) \cap N_{\varepsilon}\left(S_{H}\right)$ then $\gamma \in \Gamma_{H}$.

Proof. If not, there are $\gamma_{n} \in \Gamma$ not in $\Gamma_{H}$ and $z_{n} \in S$ such that $z_{n}, \gamma_{n} z_{n} \in N_{\varepsilon_{n}}\left(S_{H}\right)$ with $\varepsilon_{n} \rightarrow 0$. Let $y_{n}=\pi\left(z_{n}\right), y_{n}^{\prime}=\pi\left(\gamma_{n} z_{n}\right)$. Let $\lambda_{n}, \lambda_{n}^{\prime} \in \Gamma_{H}$ be such that $\lambda_{n} y_{n}, \lambda_{n}^{\prime} \lambda y_{n}^{\prime} \in \mathcal{F}_{H} / K_{H}$. Then replacing $z_{n}$ with $\lambda_{n} z_{n}$ and $\gamma_{n}$ with $\lambda_{n}^{\prime} \lambda_{n} \gamma_{n} \lambda_{n}^{-1}$ we may assume that $y_{n}, y_{n}^{\prime} \in \mathcal{F}_{H} / K_{H}$ 
(and still $\gamma_{n} \notin \Gamma_{H}$ ). Passing to a subsequence we may assume that $y_{n} \rightarrow y_{\infty}, y_{n}^{\prime} \rightarrow y_{\infty}^{\prime}$. We also have $d\left(z_{n}, y_{n}\right)=d\left(\gamma_{n} z_{n}, y_{n}^{\prime}\right) \leq \varepsilon_{n} \rightarrow 0$ so also $z_{n} \rightarrow y_{n}, \gamma_{n} z_{n} \rightarrow y_{n}^{\prime}$. In particular $\gamma_{n}$ move $y_{\infty}$ a bounded amount so it belongs to a finite set, and we may further assume the sequence is a constant element $\gamma$ such that $\gamma y_{n}=y_{n}^{\prime}$. But then $\gamma \in \Gamma \cap H K$ so $\gamma \in \Gamma_{H}$ as claimed.

Corollary 2.5 (Collar Neighborhood). For $\varepsilon$ small enough, $N_{\varepsilon}(M)=\Gamma_{H} \backslash N_{\varepsilon}\left(S_{H}\right)$.

2.2. Hyperbolic space. Suppose now $G=O(n, 1) \supset O(m, 1)=H$ and write $r=n-m$. For $S$ take the upper half-space model with coordinates

$$
\left(y, w_{1}, \ldots, w_{m-1}, w_{m}, \ldots, w_{n-1}\right)=\left(y, w^{\prime}, w^{\prime \prime}\right)
$$

in which $S_{H}=\left\{\left(y, w^{\prime}, 0\right)\right\}$. Here $w^{\prime}=\left(w_{1}, \ldots, w_{m-1}\right)$ and $w^{\prime \prime}=\left(w_{m}, \ldots, w_{n-1}\right)$.

Given a point $z=\left(y, w^{\prime}, w^{\prime \prime}\right) \in S$ let $\pi(z) \in S_{H}$ be the nearest-neighbor projection. Writing $w^{\prime \prime} \in \mathbb{R}^{r}$ in spherical coordinates $\left(\left|w^{\prime \prime}\right|, \Omega\right)$ with $\Omega=\frac{1}{\left|w^{\prime \prime}\right|} w^{\prime \prime}$, the projection $\pi(z)$ is the point $\left(\eta, w^{\prime}\right)$ where $\eta=\sqrt{y^{2}+\left|w^{\prime \prime}\right|^{2}}$. Writing $x=\frac{1}{y} w^{\prime \prime}$ with magnitude $|x|=\frac{\left|w^{\prime \prime}\right|}{y}$, the hyperbolic distance between $z$ and $\pi(z)$ is

$$
\rho=\rho(z, \pi(z))=\operatorname{arcsinh}|x|=\log \left(|x|+\sqrt{1+|x|^{2}}\right) .
$$

We note that $|x|=\sinh \rho, \sqrt{1+|x|^{2}}=\cosh \rho, \eta=y \cosh \rho$. We will eventually work with the coordinate system $\left(\eta, w^{\prime}, x\right)$ but as an intermediate step also use the system $\left(\eta, w^{\prime}, \rho, \Omega\right)$ for which the inverse map to the initial coordinate system is $y=\frac{\eta}{\cosh \rho},\left|w^{\prime \prime}\right|=\eta \tanh \rho$, $w^{\prime \prime}=(\eta \tanh \rho) \Omega$.

We can now compute the Laplace operator in these coordinates, starting from the expression in the upper-halfspace coordinates $\left(y, w^{\prime}, w^{\prime \prime}\right)$

$$
\Delta_{N}=\left(y \frac{\partial}{\partial y}\right)^{2}-(n-1)\left(y \frac{\partial}{\partial y}\right)+y^{2}\left(\Delta_{w^{\prime}}+\Delta_{w^{\prime \prime}}\right)
$$

where $\Delta_{w^{\prime}}=\sum_{i} \frac{\partial^{2}}{\partial\left(w_{i}^{\prime}\right)^{2}}$ and similarly for $w^{\prime \prime}$. We note for reference that $\frac{d \rho}{d|x|}=\frac{1}{\sqrt{1+|x|^{2}}}$ so that $\frac{\partial \rho}{\partial y}=\frac{1}{\sqrt{1+|x|^{2}}} \frac{\partial|x|}{\partial y}$ and similarly for $w_{i}^{\prime \prime}$ instead of $y$.

By the collar neighborhood lemma, we may use the Fermi normal coordinate system (established above for $S$ globally) locally in $N_{\varepsilon}(M)$, therefore giving us:

LEMMA 2.6. Using the coordinate system $\left(\eta, w^{\prime}, x\right)$ in the collar neighborhood described in (2.5), the Laplace-Beltrami operator has the following Fermi-normal coordinate-type structure:

$$
\Delta_{N}=\frac{1}{1+|x|^{2}} \Delta_{M}+\Delta_{x}+\left(\sum_{i=1}^{r} x_{i} \frac{\partial}{\partial x_{i}}\right)^{2}+(n-1)\left(\sum_{i=1}^{r} x_{i} \frac{\partial}{\partial x_{i}}\right)
$$

where $x \in \mathbb{R}^{r}$. Furthermore, the volume element on $N$ in these coordinates is

$$
\left(\eta^{-m} d \eta d^{m-1} w^{\prime}\right)\left(\left(1+|x|^{2}\right)^{\frac{m-1}{2}} d^{r} x\right),
$$

where the first term is the volume element of the hyperbolic metric on $M$. 
Proof. We give only the main parts of the calculation and leave the intermediate steps to the reader.

We have

$$
\frac{\partial}{\partial y}=\frac{1}{\cosh \rho} \frac{\partial}{\partial \eta}-\frac{1}{y} \tanh \rho \frac{\partial}{\partial \rho}
$$

Using $y=\frac{\eta}{\cosh \rho}$ gives

$$
y \frac{\partial}{\partial y}=\frac{1}{\cosh ^{2} \rho} H-(\tanh \rho) R
$$

where we have set $H=\eta \frac{\partial}{\partial \eta}$ and $R=\frac{\partial}{\partial \rho}$. Similarly we have

$$
\frac{\partial}{\partial\left|w^{\prime \prime}\right|}=\frac{1}{\eta}((\tanh \rho) H+R) \text {. }
$$

Now,

$$
\left(y \frac{\partial}{\partial y}\right)^{2}=\frac{1}{\cosh ^{4} \rho} H^{2}+\frac{2 \sinh ^{2} \rho}{\cosh ^{4} \rho} H-\frac{2 \sinh \rho}{\cosh ^{3} \rho} H R+\frac{\sinh ^{2} \rho}{\cosh ^{2} \rho} R^{2}+\frac{\sinh \rho}{\cosh ^{3} \rho} R
$$

and

$$
\left(\frac{\partial}{\partial\left|w^{\prime \prime}\right|}\right)^{2}=\frac{1}{\eta^{2}}\left(\frac{\sinh ^{2} \rho}{\cosh ^{2} \rho} H^{2}+\frac{1-\sinh ^{2} \rho}{\cosh ^{2} \rho} H+\frac{2 \sinh \rho}{\cosh \rho} H R+R^{2}-\frac{\sinh \rho}{\cosh \rho} R\right) .
$$

Finally, the Euclidean Laplace operator in polar coordinates reads

$$
\Delta_{w^{\prime \prime}}=\frac{\partial^{2}}{\left(\partial\left|w^{\prime \prime}\right|\right)^{2}}+\frac{r-1}{\left|w^{\prime \prime}\right|} \frac{\partial}{\partial\left|w^{\prime \prime}\right|}+\frac{1}{\left|w^{\prime \prime}\right|^{2}} \Delta_{S^{r-1}}
$$

A tedious calculation then gives for the coordinate system $\left(\eta, w^{\prime} ; \rho, \Omega\right)$

$$
\Delta_{N}=\frac{1}{\cosh ^{2} \rho} \Delta_{M}+F+\frac{1}{\sinh ^{2} \rho} \Delta_{S^{r-1}},
$$

where

$$
\Delta_{M}=\left(\eta \frac{\partial}{\partial \eta}\right)^{2}-(m-1)\left(\eta \frac{\partial}{\partial \eta}\right)+\eta^{2} \Delta_{w^{\prime}}
$$

is the Laplace-Beltrami operator of $S_{H}$ and

$$
F=R^{2}+\left((n-1) \tanh \rho+\frac{r-1}{\sinh \rho \cosh \rho}\right) R .
$$

We now replace $(\rho, \Omega)$ with $(|x|, \Omega)$. Using $\frac{\partial}{\partial \rho}=\sqrt{1+|x|^{2}} \frac{\partial}{\partial|x|}$ leads to

$$
F+\frac{1}{\sinh ^{2} \rho} E=\Delta_{x}+\left(\sum_{i=1}^{r} x_{i} \frac{\partial}{\partial x_{i}}\right)^{2}+(n-1)\left(\sum_{i=1}^{r} x_{i} \frac{\partial}{\partial x_{i}}\right)
$$

where $\Delta_{x}$ is the Euclidean Laplacian. Here, we have used that $|x| \frac{\partial}{\partial|x|}=\sum_{i=1}^{r} x_{i} \frac{\partial}{\partial x_{i}}$ after changing from polar coordinates back to Cartesian. 


\section{Semiclassical PRELIminaries}

In this section we recall the concepts and definitions from semiclassical analysis required for the sequel. Notations are drawn from the monographs [32].

3.1. Pseudodifferential operators on a manifold. Recall the standard classes of symbols on $\mathbb{R}^{2 d}$

$$
S^{m}\left(\mathbb{R}^{2 d}\right) \stackrel{\text { def }}{=}\left\{a \in C^{\infty}\left(\mathbb{R}^{2 d} \times(0,1]\right):\left|\partial_{x}^{\alpha} \partial_{\xi}^{\beta} a\right| \leq C_{\alpha, \beta}\langle\xi\rangle^{m-|\beta|}\right\} .
$$

Symbols in this class can be quantized through the $\hbar$-Weyl quantization into the following pseudodifferential operators acting on $u \in \mathcal{S}\left(\mathbb{R}^{d}\right)$ :

$$
\mathrm{Op}_{\hbar}^{\mathrm{w}}(a) u(x) \stackrel{\text { def }}{=} \frac{1}{(2 \pi \hbar)^{d}} \int_{\mathbb{R}^{2 d}} e^{\frac{i}{\hbar}\langle x-y, \xi\rangle} a\left(\frac{x+y}{2}, \xi ; \hbar\right) u(y) d y d \xi .
$$

One can adapt this quantization procedure to the case of the phase space $T^{*} M$, where $M$ is a smooth compact manifold of dimension $d$ (without boundary) by performing the Fourier analysis in local coordinates.

In more detail, cover $M$ with a finite set $\left(f_{l}, V_{l}\right)_{l=1, \ldots, L}$ of coordinate charts, where each $f_{l}$ is a smooth diffeomorphism from $V_{l} \subset M$ to a bounded open set $W_{l} \subset \mathbb{R}^{d}$. To each $f_{l}$ correspond a pullback $f_{l}^{*}: C^{\infty}\left(W_{l}\right) \rightarrow C^{\infty}\left(V_{l}\right)$ and a symplectic diffeomorphism $\tilde{f}_{l}$ from $T^{*} V_{l}$ to $T^{*} W_{l}$ :

$$
\tilde{f}_{l}:(x, \xi) \mapsto\left(f_{l}(x),\left(D f_{l}(x)^{-1}\right)^{T} \xi\right) .
$$

Choose a smooth partition of unity $\left(\varphi_{l}\right)_{l}$ adapted to our cover. That means $\sum_{l} \varphi_{l}=1$ and $\varphi_{l} \in C^{\infty}\left(V_{l}\right)$. Then, any observable $a$ in $C^{\infty}\left(T^{*} M\right)$ can be decomposed as $a=\sum_{l} a_{l}$, where $a_{l}=a \varphi_{l}$. Each $a_{l}$ belongs to $C^{\infty}\left(T^{*} V_{l}\right)$ and can be identified with the function $\tilde{a}_{l}=\left(\tilde{f}_{l}^{-1}\right)^{*} a_{l} \in C^{\infty}\left(T^{*} W_{l}\right)$. We now define the class of symbols of order $m$ on $T^{*} M$ (slightly abusing notation by treating $(x, \xi)$ as coordinates on $\left.T^{*} W_{l}\right)$ via

$$
\begin{aligned}
S^{m}\left(T^{*} M\right) \stackrel{\text { def }}{=} & \left\{a \in C^{\infty}\left(T^{*} M \times(0,1]\right): a=\sum_{l} a_{l},\right. \text { such that } \\
& \left.\tilde{a}_{l} \in S^{m}\left(\mathbb{R}^{2 d}\right) \text { for each } l\right\} .
\end{aligned}
$$

This class is independent of the choice of cover and partition of unity. For any $a \in S^{m}\left(T^{*} M\right)$, one can associate to each component $\tilde{a}_{l} \in S^{m}\left(\mathbb{R}^{2 d}\right)$ its Weyl quantization $\mathrm{Op}_{\hbar}^{\mathrm{w}}\left(\tilde{a}_{l}\right)$, which acts on functions on $\mathbb{R}^{2 d}$. To get back to operators acting on $M$, we choose smooth cutoffs $\psi_{l} \in C_{\mathrm{c}}^{\infty}\left(V_{l}\right)$ such that $\psi_{l}=1$ close to the support of $\varphi_{l}$, and set

$$
\mathrm{Op}_{\hbar}(a) u \stackrel{\text { def }}{=} \sum_{l} \psi_{l} \times\left(f_{l}^{*} \mathrm{Op}_{\hbar}^{\mathrm{w}}\left(\tilde{a}_{l}\right)\left(f_{l}^{-1}\right)^{*}\right)\left(\psi_{l} \times u\right), \quad u \in C^{\infty}(M) .
$$

This quantization procedure maps (modulo smoothing operators with seminorms $\mathcal{O}\left(\hbar^{\infty}\right)$ ) symbols $a \in S^{m}\left(T^{*} M\right)$ onto the space $\Psi_{\hbar}^{m}(M)$ of semiclassical pseudodifferential operators of order $m$. The dependence in the cutoffs $\varphi_{l}$ and $\psi_{l}$ only appears at order $\hbar \Psi_{\hbar}^{m-1}$ (Theorem 18.1 .17 [20] or Theorem 9.10 [32]), so that the principal symbol map $\sigma_{0}: \Psi_{\hbar}^{m}(M) \rightarrow$ $S^{m}\left(T^{*} M\right) / \hbar S^{m-1}\left(T^{*} M\right)$ is intrinsically defined. Most of microlocal calculus on $\mathbb{R}^{d}$ (for example the composition of operators, the Egorov and Calderón-Vaillancourt Theorems) then extends to the manifold case. 
An important example of a pseudodifferential operator is the semiclassical Laplace-Beltrami operator $P(\hbar)=-\frac{\hbar^{2}}{2} \Delta_{g}$. In local coordinates $(x ; \xi)$ on $T^{*} M$, the operator can be expressed as $\mathrm{Op}_{\hbar}^{\mathrm{w}}\left(|\xi|_{g}^{2}+\hbar\left(\sum_{j} b_{j}(x) \xi_{j}+c(x)\right)+\hbar^{2} d(x)\right)$ for some functions $b_{j}, c, d$ on $M$. In particular, its semiclassical principal symbol is the function $|\xi|_{g}^{2} \in S^{2}\left(T^{*} M\right)$. Similarly, the principal symbol of the Schrödinger operator $-\frac{\hbar^{2}}{2} \Delta_{g}+V(x)$ (with $\left.V \in C^{\infty}(M)\right)$ is $|\xi|_{g}^{2}+V(x) \in S^{2}\left(T^{*} M\right)$.

We will need the slightly more general class of symbols used in [14] (in fact, its adapation to $T^{*} M$ via the partition of unity argument above). In Euclidean space this is the class given for any $0 \leq \delta<1 / 2$ by

$$
S_{\delta}^{m}\left(\mathbb{R}^{2 d}\right) \stackrel{\text { def }}{=}\left\{a \in C^{\infty}\left(\mathbb{R}^{2 d} \times(0,1]\right):\left|\partial_{x}^{\alpha} \partial_{\xi}^{\beta} a\right| \leq C_{\alpha, \beta} \hbar^{-\delta|\alpha+\beta|}\langle\xi\rangle^{m-|\beta|}\right\} .
$$

These symbols are allowed to oscillate more strongly when $\hbar \rightarrow 0$. All the previous remarks regarding the case of $\delta=0$ transfer over in a straightforward manner.

\section{Ansatz and Quantum Birkhoff Normal Forms}

Let us remind ourselves that the variable $x \in \mathbb{R}^{r}$ is transverse to our submanifold $M$ and that our construction will be a quasimode which localizes in this variable.

4.1. Separation of variables and the derivation of a hyperbolic operator. At this point, we must fix our Fermi collar neighborhood (2.5) and therefore fix its corresponding width $\varepsilon_{1}>0$ (the subscript is intentional, for reasons to be explained later). In spite of our construction being entirely local, we will later show that it extends globally therefore making our norm estimates and spectral-width estimates independent of the chosen neighborhood $N_{\varepsilon_{1}}(M)$, after choosing our spectral parameters $\hbar \leq \hbar_{0}\left(M, \varepsilon_{1}\right)$

For the problem of finding sufficiently thin spectral-width quasimodes whose semiclassical measures place mass on the energy surface $S_{E_{0}} M$, we consider the following natural ansatz in our coordinate system of Lemma 2.6:

$$
\Psi_{\hbar}\left(\eta, w^{\prime}, x\right)=\Psi_{\hbar}(x) \varphi_{\hbar}\left(\eta, w^{\prime}\right) .
$$

For the moment we choose $\varphi_{\hbar}$ to be an exact eigenfunction of $\Delta_{M}$, in that $-\hbar^{2} \Delta_{M} \varphi_{\hbar}=$ $E_{0} \varphi_{\hbar}$ for some $E_{0}>0$; hence, the problem is to then choose $\Psi_{\hbar}(x)$ appropriately. Our energies will have the form $E_{\hbar}=E_{0}+f(\hbar)$, where $f(\hbar)=\mathcal{O}(\hbar)$, so we need to consider the quantity

$$
\left(-\hbar^{2} \Delta_{N}-E_{\hbar}\right) \Psi_{\hbar}\left(\eta, w^{\prime}, x\right)=0
$$

whilst keeping in mind the operator from Lemma 2.6. The above expression then takes the form

$$
\left[-\hbar^{2}\left(\Delta_{x}+\left(\sum_{i=1}^{r} x_{i} \frac{\partial}{\partial x_{i}}\right)^{2}+(n-1)\left(\sum_{i=1}^{r} x_{i} \frac{\partial}{\partial x_{i}}\right)\right)-\frac{E_{0}|x|^{2}}{1+|x|^{2}}-f(\hbar)\right] \Psi_{\hbar}(x) \varphi_{\hbar}\left(\eta, w^{\prime}\right)=0 .
$$

We therefore isolate the $x$-variable operator

$$
K_{x}(\hbar) \stackrel{\text { def }}{=}-\hbar^{2}\left(\Delta_{x}+\left(\sum_{i=1}^{r} x_{i} \frac{\partial}{\partial x_{i}}\right)^{2}+(n-1)\left(\sum_{i=1}^{r} x_{i} \frac{\partial}{\partial x_{i}}\right)\right)-\frac{E_{0}|x|^{2}}{1+|x|^{2}}-f(\hbar) .
$$


The factorization of the volume element in Lemma 2.6 shows we should consider $K_{x}(\hbar)$ acting on $L^{2}\left(\mathbb{R}^{r},\left(1+|x|^{2}\right)^{\frac{m-1}{2}} d x\right)$, where it will be a formally symmetric operator (on smooth compactly supported functions) by virtue of $\Delta_{N}$ being symmetric.

LEMma 4.5. Suppose that $\left\{\varphi_{\hbar}\right\}_{\hbar}$ are $L^{2}(M)$-normalized quasimodes for $-\hbar^{2} \Delta_{M}$, with central energy $E_{0}$ and of width $\frac{c_{1} \hbar}{|\log \hbar|}$, converging to the semiclassical measure $\mu_{s c}$ on $S_{E_{0}} M$. Suppose that $\left\{\Psi_{\hbar}\right\}_{\hbar}$ are $L^{2}\left(\mathbb{R}^{r},\left(1+|x|^{2}\right)^{(m-1) / 2}\right)$-normalized quasimodes for $K_{x}(\hbar)$ supported in the collar $B\left(0, \varepsilon_{1}\right)$ with central energy 0 and of width $\frac{c_{2} \hbar}{|\log \hbar|}$, converging to a semiclassical measure $\sigma_{s c}$ on $T^{*}\left(\mathbb{R}^{r}\right)$ such that $\sigma_{s c} \geq \nu \delta_{0}$; in other words, $\sigma$ gives mass at least $\nu \in(0,1]$ to the point $(0 ; 0)$.

Then $\left\{\boldsymbol{\Psi}_{\hbar}\right\}_{\hbar}$ are normalized quasimodes for $-\hbar^{2} \Delta_{N}$ with central energies $E_{\hbar}=E_{0}+f(\hbar)$ of width $\frac{\left(c_{1}+c_{2}\right) \hbar}{|\log \hbar|}$, converging to a semiclassical measure $\tilde{\mu}_{s c}$ giving mass at least $\nu$ to $\iota_{*} \mu_{s c}$ where $\iota: M \rightarrow N$ is the inclusion map. In particular when $\nu=1$ the quantum limit is exactly $\iota_{*} \mu_{s c}$.

Proof. Since our collar $N_{\varepsilon_{1}}(M)$ factors as a product $B\left(0, \varepsilon_{1}\right) \times M$ in the coordinate system, the identification of the limit is clear. It remains to verify that $\left\{\boldsymbol{\Psi}_{\hbar}\right\}_{\hbar}$ are indeed quasimodes of the stated width. Following the calculation above and Lemma 2.6, we have:

$$
\begin{aligned}
& \left(-\hbar^{2} \Delta_{N}-E_{\hbar}\right) \boldsymbol{\Psi}_{\hbar}=\left(\frac{-1}{1+|x|^{2}} \hbar^{2} \Delta_{M}+K_{x}(\hbar)+\frac{E_{0}|x|^{2}}{1+|x|^{2}}+f(\hbar)\right) \boldsymbol{\Psi}_{\hbar} \\
& \left(K_{x}(\hbar) \Psi_{\hbar}\right)(x) \varphi_{\hbar}\left(\eta, w^{\prime}\right)+\Psi_{\hbar}(x) \frac{1}{1+|x|^{2}}\left(-\hbar^{2} \Delta_{M}-E_{\hbar}\right) \varphi_{\hbar}\left(\eta, w^{\prime}\right)
\end{aligned}
$$

Now by assumption,

$$
\begin{gathered}
\left\|K_{x}(\hbar) \Psi_{\hbar}\right\|_{L^{2}\left(\mathbb{R}^{r},\left(1+|x|^{2}\right)^{\frac{m-1}{2}} d x\right)} \leq \frac{c_{2} \hbar}{|\log \hbar|}, \\
\left\|\frac{1}{1+|x|^{2}} \Psi_{\hbar}(u)\right\|_{L^{2}\left(\mathbb{R}^{r},\left(1+|x|^{2}\right)^{\frac{m-1}{2}} d x\right)} \leq\left\|\Psi_{\hbar}(x)\right\|_{L^{2}\left(\mathbb{R}^{r},\left(1+|x|^{2}\right)^{\frac{m-1}{2}} d x\right)}=1,
\end{gathered}
$$

and

$$
\begin{aligned}
& \left\|\frac{1}{1+|x|^{2}}\left(-\hbar^{2} \Delta_{M}-E_{\hbar}\right) \varphi_{\hbar}\left(\eta, w^{\prime}\right) \Psi_{\hbar}\right\|_{L^{2}(N)}= \\
& \left\|\frac{1}{1+|x|^{2}} \Psi_{\hbar}\right\|_{L^{2}\left(\mathbb{R}^{r},\left(1+|x|^{2}\right)^{(m-1) / 2}\right)}\left\|\left(-\hbar^{2} \Delta_{M}-E_{\hbar}\right) \varphi_{\hbar}\left(\eta, w^{\prime}\right)\right\|_{L^{2}(M)} \leq \frac{c_{1} \hbar}{|\log \hbar|},
\end{aligned}
$$

together giving the claim after applying Fubini's theorem thanks to the product form of the metric in Lemma 2.6.

For the rest of the paper we will then construct transverse-to- $M$ quasimodes $\Psi_{\hbar}$ of central energy 0 for the operator $K_{x}(\hbar)$, such that the quasimodes microlocalize to the delta measure $\delta_{0}(x)$ or at least to a semiclassical measure containing this measure. 
Writing $\xi$ for the variable dual to $u$ on $\mathbb{R}^{r}$, the total symbol of $K_{x}(\hbar)$ as an $\hbar$-pseudodifferential operator (see Section 3) is

$$
|\xi|^{2}+(x \cdot \xi)^{2}+(n-1) \hbar(x \cdot \xi)-\frac{E_{0}|x|^{2}}{1+|x|^{2}}-f(\hbar)
$$

hence giving a semiclassical principal symbol of

$$
\sigma(x ; \xi)=|\xi|^{2}+(x \cdot \xi)^{2}-\frac{E_{0}|x|^{2}}{1+|x|^{2}} ;
$$

the reader is referred to [32] for further details on the symbol map. Observe that that $\sigma$ differs from $\tilde{\sigma}:=|\xi|^{2}-E_{0}|x|^{2}$, the principal symbol for the inverted harmonic oscillator, by terms of order $\mathcal{O}(|x|+|\xi|)^{4}$ when $(x ; \xi)$ is sufficiently close to $(0 ; 0)$. It follows that that $\sigma$ retains the non-degenerate critical point of $\tilde{\sigma}$ at $(0 ; 0)$. The expression $\tilde{\sigma}$ is a split quadratic form with eigenvalues $\left\{ \pm \mu_{i}\right\}_{i=1}^{r}$ where $\mu_{i}>0$. A linear change of variables along with $\xi \rightarrow \sqrt{E_{0}} \xi$ transforms $\tilde{\sigma}$ to $\sum_{i=1}^{r} 2 \sqrt{E_{0}} x_{i} \xi_{i}$ whose Hamiltonian flow is the model for a hyperbolic fixed point.

We will obtain our quasimodes $\Psi_{\hbar}$ from the following "quantized" version of a classical Birkhoff normal form (see the beautiful text [1] by Abraham-Marsden for more on this topic) specifically due to Iantchenko [21] but inspired by the work of Sjöstrand [27] in the context of Hamiltonians with non-degenerate minima. We state it in a slightly different fashion which is more adapted to our upcoming calculations:

Proposition 4.14. Let $P(\hbar)$ be a formally self-adjoint pseudodifferential operator, or rather a symmetric operator, acting on $C_{\mathrm{c}}^{\infty}\left(\mathbb{R}^{r}\right)$ with a $L^{2}\left(\mathbb{R}^{r}, \mu\right)$ structure for some positive measure $\mu$. Consider its real Weyl symbol $\sum_{j=0}^{\infty} \hbar^{j} p_{j}$. Suppose further that its semiclassical principal symbol $p_{0}$ has a non-degenerate critical point and defines a split quadratic form. That is $p(0 ; 0)=0, d p(0 ; 0)=0$, and $p^{\prime \prime}(0 ; 0)$ is non-degenerate but whose eigenvalues are $\left\{ \pm \frac{\lambda_{i}}{2}\right\}_{i=1}^{r}$ where $\lambda_{i}>0$. Set $\vec{\lambda} \stackrel{\text { def }}{=}\left(\lambda_{1}, \ldots, \lambda_{r}\right)$.

For $\tilde{N}>0$ given, we can find neighborhoods $U, V$ of $(0 ; 0)$ in $T^{*} \mathbb{R}^{r}$ and a canonical transformation $\kappa: U \rightarrow V$, with $\kappa(0 ; 0)=(0 ; 0)$, such that

$$
\left(\sum_{j=0}^{\tilde{N}} \hbar^{j} p_{j}\right) \circ \kappa=q^{(\tilde{N})}+r_{N+1}=\sum_{j=0}^{\tilde{N}} \hbar^{j} q_{j}(x ; \xi)+r_{\tilde{N}+1}(x ; \xi ; \hbar),
$$

where

$$
q_{0}=\sum_{i=1}^{r} \lambda_{i} x_{i} \xi_{i}+\sum_{l=2}^{\tilde{N}}\left(\sum_{\alpha-\beta \in \mathcal{M},|\alpha|+|\beta|=l} c_{\alpha, \beta} x^{\alpha} \xi^{\beta}\right) .
$$

Here $\mathcal{M}=\left\{\gamma \in \mathbb{N}^{n}: \vec{\lambda} \cdot \gamma=0\right\}$ is the so-called resononance module for the Hamiltonian $q_{0,1}=\sum_{i} \lambda_{i} x_{i} \xi_{i}$ and $r_{\tilde{N}+1}(x ; \xi ; \hbar)=\mathcal{O}\left((\hbar+|x|+|\xi|)^{\tilde{N}+1}\right)$. The other terms $q_{j}$ have a similar form and for each $j, H_{q_{0,1}} q_{j}=0$. Here, $H_{q_{0,1}}$ is the Hamiltonian vector field associated to the function $q_{0,1}$ and takes the form $x \cdot \frac{\partial}{\partial x}-\xi \cdot \frac{\partial}{\partial \xi}$.

Moreover, we have a corresponding quantum Birkhoff normal form. That is, for the given $\tilde{N}$ and $U, V$ as above there exists a microlocally unitary semiclassical Fourier integral operator $U(h): C_{\mathrm{c}}^{\infty}\left(\mathbb{R}^{r}\right) \rightarrow \mathcal{S}\left(\mathbb{R}^{r}\right)$ near $(0 ; 0)$. In other words, with respect to the given $L^{2}$ 
structure, $\|U(\hbar)\|_{L^{2}}=\left\|\chi_{2}\left(x ; \hbar D_{x}\right) U(\hbar) \chi_{1}\left(x ; \hbar D_{x}\right)\right\|_{L^{2}}+\mathcal{O}_{L^{2}}\left(\hbar^{\infty}\right)$ for all microlocal cutoffs $\chi_{1}, \chi_{2}$ supported within $U$ and $V$ respectively and

$$
U_{\tilde{N}}^{*}(\hbar) P(\hbar) U_{\tilde{N}}(\hbar)=Q^{(\tilde{N})}(\hbar)+R^{(\tilde{N}+1)}(\hbar) .
$$

Here, $Q^{(\tilde{N})}(\hbar)$ and $R^{(\tilde{N}+1)}(\hbar)$ are semiclassical pseudodifferential operators with microlocal support in the indicated neighborhoods above with respective Weyl symbols $q^{(\tilde{N})}$ and $r_{\tilde{N}+1}$ where $\left[\mathrm{Op}_{\hbar}^{w}\left(\sum_{i} \lambda_{i} x_{i} \xi_{i}\right), Q^{(\tilde{N})}(\hbar)\right]=0$.

Remark 4.18. In our setting where the energy $E=E_{0}$, the corresponding fixed point $(0 ; 0)$ for our pre-normal form symbol in (4.13) yields expansion rates $\lambda_{i}=2 \sqrt{E_{0}}$ after the discussed scaling. For the sake of simplicity within our upcoming calculations and as the maximal Lyapunov exponent $\tilde{\lambda}_{E_{0}}$ on the energy shell $S_{E_{0}}^{*} N$ scales as $\sqrt{E_{0}} \tilde{\lambda}_{1}$, we consider the case of $\sqrt{E_{0}}=1$ for the remainder of our article. We make note that the expansion rates transverse to $M$ are equal across the entire ambient manifold $N$, which is a feature of the constant curvature setting.

\section{Propagation of a Gaussian wavepacket at the Hyperbolic Fixed Point}

We begin this section with the following important remark:

Remark 5.1. This Section gives the higher-dimensional analogues of the results in Section 5 of [15]. The arguments are very similar, but we give considerable detail for two reasons. First, while the $r$-dimensional inverted harmonic oscillator obviously factors into a product of $r$ one-dimensional oscillators (for initial conditions which factor), the quantum Birkhoff normal form includes additional error terms, and we need to track their behavior after conjugating by the semiclassical FIO in Proposition 4.14. Second, the argument for Corollary 1.10 differs from previous quasimode constructions in that we vary the submanifold $M$. Accordingly we must ensure that the spectral width, particularly the parameter $C>0$, of our quasimodes does not depend on the submanifold $M$.

\subsection{Ground states, squeezed states, and evolution.}

\subsubsection{Preparing the Hamiltonian. Consider the Gaussian}

$$
\Phi_{0}(x) \stackrel{\text { def }}{=} \frac{1}{(\pi \hbar)^{r / 4}} \prod_{i=1}^{r} \exp \left(-\frac{x_{i}^{2}}{2 \hbar}\right)
$$

which is the $L^{2}$-normalized ground state of the harmonic oscillator on $\mathbb{R}^{r}$ and has width $\hbar^{1 / 2}$ in each direction $x_{i}$. We will evolve $\Phi_{0}$ through the reduced time-dependent Schrödinger equation generated by the QNF operator $Q^{(\tilde{N})}$ described in Proposition 4.14, i.e. given the problem

$$
\begin{cases}i \hbar \partial_{t} \Phi_{t}^{(\tilde{N})} & =Q^{(\tilde{N})}(\hbar) \Phi_{t}^{(\tilde{N})}, \text { where } Q^{(\tilde{N})}=\mathrm{Op}_{\hbar}^{\mathrm{w}}\left(q^{(\tilde{N})}\right) \\ \Phi_{t \mid t=0}^{(\tilde{N})} & =\Phi_{0},\end{cases}
$$

we must analyze the evolution of $\Phi_{0}(x)$. Therefore, our goal in this section is to describe the states

$$
\Phi_{t}^{(\tilde{N})}=e^{-i Q^{(\tilde{N})} t / \hbar} \Phi_{0} \quad \text { for times }|t| \leq C\left(\tilde{\lambda}_{1}, \varepsilon_{2}\right)|\log \hbar|
$$


where $C>0$ will be an explicit constant depending only on the maximal Lyapunov exponent $\tilde{\lambda}_{1}$ of $M$, in this case being 2 , and a small parameter $\varepsilon_{2}$. The index $\tilde{N}$ will be chosen later.

The Weyl symbol of $Q^{(\tilde{N})}$ arising from Proposition 4.14 can be rewritten as

$$
q^{(\tilde{N})}(x, \xi ; h)=\left(\sum_{i=1}^{r} q_{1, i}(h) x_{i} \xi_{i}\right)+\sum_{j=2}^{\tilde{N}}\left(\sum_{|\alpha|=|\beta|=j} q_{j}^{\alpha, \beta}(\hbar) x^{\alpha} \xi^{\beta}\right),
$$

where the coefficients $q_{j}^{\alpha, \beta}(\hbar)$ expand like

$$
q_{j}^{\alpha, \beta}(\hbar)=\sum_{i=0}^{\tilde{N}} \hbar^{i} q_{j, i}^{\alpha, \beta}, \text { for } q_{j, i}^{\alpha, \beta} \text { a constant }
$$

for $j \geq 2$. Moreover, for $j=1, q_{1, i}(h)=\lambda_{i}+\mathcal{O}(h)$. Here, we have used that $\lambda_{i}=2$ for all $i$ and thus that the resonance module is $\mathcal{M}=\left\{\gamma \in \mathbb{N}^{r}: \sum_{i} \gamma_{i}=0\right\}=\{0\}$. The top order terms of the coefficients $q_{1, i}$ will play an important role in the Schrödinger evolution around the unstable fixed point $(0 ; 0)$. In order to make the following analysis more transparent, we will truncate our Weyl symbol $q^{(\tilde{N})}$ to its top order quadratic terms and set

$$
q_{\text {quad }}=\sum_{i=1}^{r} \lambda_{i} x_{i} \xi_{i}
$$

We label the operator whose Weyl symbol is $q_{\text {quad }}$ as $Q_{\text {quad }}(h)$. Notice that this operator and its full symbol are independent of $\tilde{N}$.

Recall from Proposition 4.14 that $\vec{\lambda}=\left(\lambda_{1}, \ldots, \lambda_{r}\right)$. The Schrödinger propagator for this special quadratic operator is easily expressed as

$$
U_{\text {quad }}(t) \Phi_{0}:=\exp \left(-\frac{i t}{\hbar} Q_{\text {quad }}\right) \Phi_{0}=\mathcal{D}_{t \rightarrow} \Phi_{0},
$$

where the unitary dilation operator $\mathcal{D}_{\vec{\lambda}}: L^{2}\left(\mathbb{R}^{r}\right) \rightarrow L^{2}\left(\mathbb{R}^{r}\right)$ is given by

$$
\mathcal{D}_{\vec{\lambda}} u(x) \stackrel{\text { def }}{=} \exp \left(-i\left[\sum_{i} \lambda_{i} \mathrm{Op}_{\hbar}^{\mathrm{w}}\left(x_{i} \xi_{i}\right)\right] / \hbar\right) u(x)=e^{-\sum_{i} \lambda_{i} / 2} u\left(e^{-\lambda_{1}} x_{1}, \ldots, e^{-\lambda_{r}} x_{r}\right) .
$$

The states $\mathcal{D}_{\vec{\lambda}} \Phi_{0}(x)$ and their generalizations are known as squeezed states. They naturally appear in related problems. Two applications include the Gutzwiller trace formula [13] and the pioneering work of Babich-Lazutkin [22] on the construction of quasimodes concentrating on closed elliptic geodesics. For more applications of these special states see [13] and the references therein.

For $\tilde{N} \geq 2$, the operator $Q^{(\tilde{N})}$ includes a nonquadratic Hamilton $Q_{\mathrm{nq}}^{(\tilde{N})}$ with its Weyl symbol taking the form

$$
q_{\mathrm{nq}}^{(\tilde{N})} \stackrel{\text { def }}{=} q^{(\tilde{N})}-\left(\sum_{i=1}^{r} q_{1, i}(h) x_{i} \xi_{i}\right)=\sum_{j=2}^{\tilde{N}}\left(\sum_{|\alpha|=|\beta|=j} q_{j}^{\alpha, \beta}(\hbar) x^{\alpha} \xi^{\beta}\right)
$$

and

$$
\tilde{q}_{\text {quad }}=\left(\sum_{i=1}^{r} q_{1, i}(h) x_{i} \xi_{i}\right)-q_{\text {quad }}
$$


whose Weyl quantization we label as $\tilde{Q}_{\text {quad }}$. Note that as a symbol, $\tilde{q}_{\text {quad }}=\mathcal{O}(\hbar)$ and that

$$
q^{(\tilde{N})}=q_{\text {quad }}+\tilde{q}_{\text {quad }}+q_{\text {nq }}^{(\tilde{N})} .
$$

Unfortunately, the second and third terms above cannot be ignored and a large part of our construction will be in analyzing their contributions to the evolution of $\Phi_{0}$. However, the resonance condition given in Proposition 4.14 greatly simplifies the actions of $\tilde{Q}_{\text {quad }}$ and $Q_{\mathrm{nq}}^{(\tilde{N})}$ as it ensures that this operator commutes with $Q_{\text {quad }}$.

5.1.2. Squeezed excited states. We now recall some basic facts concerning the standard $r$ dimensional quantum harmonic oscillator $\sum_{i}\left(\hbar D_{x_{i}}\right)^{2}+X_{i}^{2}$ where $X_{i}$ is the operator which multiplies by $x_{i}$. Our initial state $\Phi_{0}=\prod_{i=1}^{r} \varphi_{0}\left(x_{i}\right)$ is also the ground state of this operator, where the individual functions $\varphi_{0}\left(x_{i}\right)=\frac{1}{(\pi \hbar)^{1 / 4}} e^{-\frac{x_{i}^{2}}{2 \hbar}}$ are themselves ground states of the 1-dimensional quantum harmonic oscillators $\left(\hbar D_{x_{i}}\right)^{2}+X_{i}^{2}$.

Let us call $\left(\varphi_{m}\right)_{m \geq 1}$ the 1-dimensional $m$-th excited states in the variable $x_{i}$, which are obtained by iteratively applying to $\varphi_{0}$ the "raising operator" $a_{i}^{*} \stackrel{\text { def }}{=} \mathrm{Op}_{\hbar}^{\mathrm{w}}\left(\frac{x_{i}-i \xi_{i}}{\sqrt{2 \hbar}}\right)$ and $L^{2}-$ normalizing:

$$
\varphi_{m}=\frac{\left(a^{*}\right)^{m}}{\sqrt{m !}} \varphi_{0} \Longrightarrow \varphi_{m}\left(x_{i}\right)=\frac{1}{(\pi \hbar)^{1 / 4} 2^{m / 2} \sqrt{m !}} H_{m}\left(x_{i} / \hbar^{1 / 2}\right) e^{-\frac{x_{i}^{2}}{2 \hbar}}
$$

where $H_{m}(\cdot)$ is the $m$-th Hermite polynomial. We also have the dual lowering operators $a_{i} \stackrel{\text { def }}{=} \mathrm{Op}_{\hbar}^{\mathrm{w}}\left(\frac{x_{i}+i \xi_{i}}{\sqrt{2 \hbar}}\right)$ which satisfy the similar relation $a_{i} \varphi_{m}\left(x_{i}\right)=\sqrt{m} \varphi_{m-1}\left(x_{i}\right)$.

Now, given a $r$-vector of 1-dimensional excited states $\left(\varphi_{m_{1}}, \ldots, \varphi_{m_{r}}\right)$, we can form the analogous $r$-dimensional excited state

$$
\Phi_{m_{1}, \ldots, m_{r}}(x) \stackrel{\text { def }}{=} \prod_{i=1}^{r} \varphi_{m_{i}}\left(x_{i}\right) .
$$

This function continues to have $L^{2}$-norm 1 , and by applying the unitary dilation operator $\mathcal{D}_{\vec{\lambda}}$ we obtain an $L^{2}$-normalized squeezed excited state $\mathcal{D}_{\vec{\lambda}} \Phi_{m_{1}, \ldots, m_{r}}(x)$. These are essentially products of unitarily scaled Gaussians in one variable decorated by products of scaled polynomials. The main property exhibited by $\mathcal{D}_{\vec{\lambda}} \Phi_{m_{1}, \ldots, m_{r}}$ that we will use are its concentration properties which are similar to those of $\mathcal{D}_{\vec{\lambda}} \Phi_{0}$.

5.1.3. Expansion around the fixed point. The following result is inspired by the work of Combescure-Robert [13] and its proof is an $r$-dimensional version of Proposition 4.9 in [15]. Hence, we only provide the necessary details and leave the remaining elements to the reader.

Proposition 5.13. For every $l, \tilde{N} \in \mathbb{N}$, there exists a constant $C_{l, \tilde{N}}>0$ and coefficients $c_{p}(t, \hbar) \in \mathbb{C}$, which are polynomials in $t$ and $\hbar$, such that the following estimate holds for any $\hbar \in(0,1]:$

$$
\forall t \in \mathbb{R}, \quad\left\|e^{i t Q^{(N)} / \hbar} \Phi_{0}-\mathcal{D}_{t \rightarrow \vec{\lambda}} \Phi_{0}-\sum_{p=1}^{l} c_{p}(t, \hbar) \mathcal{D}_{t \vec{\lambda}}\left(\sum_{k_{p}} d_{k_{p}} \Phi_{G\left(k_{p}\right)}\right)\right\| \leq C_{l, N}(|t| \hbar)^{l+1} .
$$


The coefficients $c_{p}(t, \hbar)$ are $\mathcal{O}\left(|t|^{l} \hbar^{p}\right)$. The integer indices $k_{p} \leq C_{p, \tilde{N}}, d_{k_{p}}>0$ are constants, and $G(p) \in \mathbb{N}^{n}$ indexes the excited states. Furthermore, the number of terms in the sums indexed by $k_{p}$ is less than some function depending only on $\tilde{N}$.

Remark 5.15. This proposition will play a crucial role in determining the microlocal concentration of our future constructed quasimode. In order to show that the spectral width of our quasimodes is uniform with respect to our submanifold $M$, we will need to keep track of the dependence of certain constants on the parameters $l$ and $\tilde{N}$. We will ultimately verify that any $l \geq 2$ is sufficient for our purposes but that $\tilde{N}$ may have to be large, specifically we shall require $(\tilde{N}+1) \varepsilon_{2} / 3>1$ where $\varepsilon_{2}$ will appear in our Ehrenfest time.

Proof. As in [13], we would like to show that the full evolved state $\Phi_{t}^{(\tilde{N})}=U(t) \Phi_{0}=$ $e^{-i Q^{(\tilde{N})} t / \hbar} \Phi_{0}$ is sufficiently approximated by $U_{\text {quad }}(t) \Phi_{0}=\mathcal{D}_{\vec{\lambda} t} \Phi_{0}(x)$, modulo a large sum of $r$-dimensional excited states whose $L^{2}$-norm is sufficiently small (bounded by an appropriate power of $\hbar$ ).

The method of approximation is via the so-called Dyson expansion of Duhamel's formula

$$
U(t)-U_{\text {quad }}(t)=\frac{1}{i h} \int_{0}^{t} U\left(t-t_{1}\right)\left(\tilde{Q}_{\text {quad }}+Q_{\text {nq }}^{(\tilde{N})}\right) U_{\text {quad }}\left(t_{1}\right) d t_{1},
$$

which corresponds to the choice $l=1$. Using this formula directly leads to the bound $\left\|U(t) \Phi_{0}-U_{\text {quad }}(t) \Phi_{0}\right\| \leq C_{1, \tilde{N}} t \hbar$ for all $t \in \mathbb{R}, \hbar \in[0,1)$ (we will later see that $t \leq$ $\left.C\left(\tilde{\lambda}_{1}, \varepsilon_{2}\right)|\log \hbar|\right)$. This is not sufficient since we want future error estimates to be $\mathcal{O}\left(h^{1+\delta}\right)$ for some $\delta>0$.

Accordingly we iterate the formula $l>1$ times to obtain

$$
\begin{aligned}
& U(t)-U_{\text {quad }}(t)= \\
& \sum_{j=1}^{l} \frac{1}{(i \hbar)^{j}} \int_{0}^{t} \int_{t_{1}}^{t} \ldots \int_{t_{j-1}}^{t} U_{\text {quad }}\left(t-t_{j}\right)\left(\tilde{Q}_{\text {quad }}+Q_{\mathrm{nq}}^{(\tilde{N})}\right) U_{\text {quad }}\left(t_{j}-t_{j-1}\right)\left(\tilde{Q}_{\text {quad }}+Q_{\mathrm{nq}}^{(\tilde{N})}\right) \\
& \quad \ldots\left(\tilde{Q}_{\text {quad }}+Q_{\mathrm{nq}}^{(\tilde{N})}\right) U_{\text {quad }}\left(t_{1}\right) d t_{1} \ldots d t_{j} \\
& +\frac{1}{(i \hbar)^{l+1}} \int_{0}^{t} \int_{t_{1}}^{t} \ldots \int_{t_{l}}^{t} U\left(t-t_{l+1}\right)\left(\tilde{Q}_{\text {quad }}+Q_{\mathrm{nq}}^{(\tilde{N})}\right) U_{\text {quad }}\left(t_{l+1}-t_{l}\right) \\
& \quad\left(\tilde{Q}_{\text {quad }}+Q_{\mathrm{nq}}^{(\tilde{N})}\right) \ldots\left(\tilde{Q}_{\text {quad }}+Q_{\mathrm{nq}}^{(\tilde{N})}\right) U_{\text {quad }}\left(t_{1}\right) d t_{1} \ldots d t_{l+1} .
\end{aligned}
$$

To simplify the notation, we shorten the last term to $R_{l}^{(N)}(t, \hbar)$.

A crucial fact involving the quantum Birkhoff normal form in Proposition 4.14 is that $\left[Q_{\mathrm{nq}}^{(\tilde{N})}, Q_{\text {quad }}^{(\tilde{N})}\right]=0$ and that $\left[\tilde{Q}_{\text {quad }}, Q_{\text {quad }}\right]=0$. This implies $Q_{\mathrm{nq}}^{(\tilde{N})}$ and $\tilde{Q}_{\text {quad }}$ also commute with $U_{\text {quad }}$ by functional calculus, effectively giving us an exact form of Egorov's Theorem when we apply the quadratic evolution to our resonant Hamiltonians: $\left(U_{\text {quad }}\right)^{*} Q_{\mathrm{nq}}^{(\tilde{N})} U_{\text {quad }}=Q_{\mathrm{nq}}^{(\tilde{N})}$ since the Weyl symbols of $Q_{\mathrm{nq}}^{(\tilde{N})}$ and $\tilde{Q}_{\text {quad }}$ are resonant functions under the action of the Hamiltonian vector field of $Q_{\text {quad }}^{(\tilde{N})}$. For proofs of Egorov's Theorem in this case, see [13, 32]. 
This commutativity leads us to the simpler expression

$$
\begin{aligned}
U(t)-U_{\text {quad }}(t)= & \sum_{p=1}^{l} \frac{t^{p}}{p !(i \hbar)^{p}} U_{\text {quad }}(t)\left(\tilde{Q}_{\text {quad }}+Q_{\text {nq }}^{(\tilde{N})}\right)^{p} \\
& +\frac{1}{(i \hbar)^{l+1}} \int_{0}^{t} \frac{t_{l}^{l}}{l !} U\left(t-t_{l+1}\right) U_{\text {quad }}\left(t_{l+1}\right)\left(\tilde{Q}_{\text {quad }}+Q_{\text {nq }}^{(\tilde{N})}\right)^{l+1} d t_{l+1} .
\end{aligned}
$$

It is helpful to understand the explicit action of $Q_{\mathrm{nq}}^{(N)}$ on our intial state $\Phi_{0}$. As

$$
((x+y) / 2)^{\alpha} \xi^{\beta}=\prod_{i=1}^{r}\left(\sum_{\gamma_{i}=0}^{\alpha_{i}}\left(\begin{array}{c}
\alpha_{i} \\
\alpha_{i}-\gamma_{i}
\end{array}\right)\left(\frac{x_{i}}{2}\right)^{\alpha_{i}-\gamma_{i}} \xi_{i}^{\beta_{i}}\left(\frac{y_{i}}{2}\right)^{\gamma_{i}}\right),
$$

we see that

$$
\mathrm{Op}_{\hbar}^{\mathrm{w}}\left(x^{\alpha} \xi^{\beta}\right)=\prod_{i=1}^{r}\left(\sum_{\gamma_{i}=0}^{\alpha_{i}}\left(\begin{array}{c}
\alpha_{i} \\
\alpha_{i}-\gamma_{i}
\end{array}\right)\left(\frac{X_{i}}{2}\right)^{\alpha_{i}-\gamma_{i}}\left(\hbar D_{x_{i}}\right)^{\beta_{i}}\left(\frac{X_{i}}{2}\right)^{\gamma_{i}}\right) .
$$

Using that $X_{i}=\sqrt{\frac{\hbar}{2}}\left(a_{i}^{*}+a_{i}\right)$ and $\hbar D_{x_{i}}=i \sqrt{\frac{\hbar}{2}}\left(a_{i}^{*}-a_{i}\right)$, where $a_{i}^{*}$ and $a_{i}$ are the raising and lowering operators in the $x_{i}$ variable, we find that (5.19) reduces to

$$
\begin{aligned}
\mathrm{Op}_{\hbar}^{\mathrm{w}}\left(x^{\alpha} \xi^{\beta}\right) & =\prod_{i=1}^{r} \mathrm{Op}_{\hbar}^{\mathrm{w}}\left(x_{i}^{\alpha_{i}} \xi_{i}^{\beta_{i}}\right) \\
& =\prod_{i=1}^{r}\left(\sum_{\gamma_{i}=0}^{\alpha_{i}}\left(\begin{array}{c}
\alpha_{i} \\
\alpha_{i}-\gamma_{i}
\end{array}\right)\left(\frac{1}{2}\right)^{\alpha_{i}}\left(\frac{\hbar}{2}\right)^{\left(\alpha_{i}+\beta_{i}\right) / 2} i^{\beta_{i}}\left(a_{i}^{*}+a_{i}\right)^{\alpha_{i}-\gamma_{i}}\left(a_{i}^{*}-a_{i}\right)^{\beta_{i}}\left(a_{i}^{*}+a_{i}\right)^{\gamma_{i}}\right) .
\end{aligned}
$$

Here we have used composition formulae for the Weyl quantization and the Moyal product for the symbols in disjoint variables. Keeping in mind the action of the raising and lowering operators on $\Phi_{0}$, it follows that

$$
\begin{aligned}
& Q_{\mathrm{nq}}^{(\tilde{N})} \Phi_{0}=\sum_{j=2}^{\tilde{N}} \sum_{|\alpha|=|\beta|=j} q_{j}^{\alpha, \beta}(\hbar) \mathrm{Op}_{\hbar}^{\mathrm{w}}\left(x^{\alpha} \xi^{\beta}\right) \Phi_{0} \\
& =\sum_{j=2}^{\tilde{N}} \sum_{|\alpha|=|\beta|=j}^{M(j, r)} q_{j}^{\alpha, \beta}(\hbar) \mathcal{O}\left(\hbar^{(|\alpha|+|\beta|) / 2}\right)\left(\prod_{i=1}^{r} \sum_{k=0}^{\left.\frac{2^{\alpha_{i}+\beta_{i}+1}}{2}\right\rceil\left(\alpha_{i}+1\right)} c_{k}^{\alpha_{i}, \beta_{i}} \varphi_{k}\left(x_{i}\right)\right) \\
& =\sum_{j=2}^{\tilde{N}} \mathcal{O}\left(\hbar^{j}\right)\left(q_{j}^{\alpha, \beta}(\hbar) \prod_{i=1}^{r} \sum_{k=0}^{\left.\frac{2^{\alpha_{i}+\beta_{i}+1}}{2}\right\rceil\left(\alpha_{i}+1\right)} c_{k}^{\alpha_{i}, \beta_{i}} \varphi_{k}\left(x_{i}\right)\right) \\
& \left.=\sum_{j=2}^{\prod_{i=1}^{r}\left\lceil\frac{2^{\alpha_{i}+\beta_{i}+1}}{\sum^{2}} \mathcal{O}\left(\alpha_{i}+1\right)\right.} c(\alpha, \beta, k) \Phi_{F(\alpha, \beta, k)}\right)
\end{aligned}
$$

where $M(j, r)=\mathcal{O}\left(P_{r}^{j}\right)$, for $P_{r}^{j}$ is the partition function, and $\Phi_{F}$ is an $r$-dimensional excited state with $F \in \mathbb{N}^{r}$ being a function of $\alpha, \beta$, and $k$. In the second line above, the inner 
sum contains at most $P_{r}^{j}$ terms and therefore gets absorbed into $\mathcal{O}\left(h^{j}\right)$ term in the next line. We have also used the fact that for odd (respectively, even) $\alpha_{i}+\beta_{i}$, the operator $\left(a_{i}^{*}+a_{i}\right)^{\alpha_{i}-\gamma_{i}}\left(a_{i}^{*}-a_{i}\right)^{\beta_{i}}\left(a_{i}^{*}+a_{i}\right)^{\gamma_{i}}$ yields a sum of odd (respectively, even) indexed excited states when applied to $\varphi_{0}\left(x_{i}\right)$. This is due to the fact that any word of length $W$ consisting of $a_{i}^{*}$ and $a_{i}$ is a sum of words in "normal ordering" $\left(a_{i}\right)^{m}\left(a_{i}^{*}\right)^{n}$ (after using that $\left[a_{i}^{*}, a_{i}\right]=1$ ) each of whose length is equal in parity to $W$. Hence, after using that $\tilde{Q}_{\text {quad }} \Phi_{0}=\mathcal{O}\left(\hbar^{2}\right)$,

$$
\left(\tilde{Q}_{\text {quad }}+Q_{\text {nq }}^{(\tilde{N})}\right)^{l} \Phi_{0}=\sum_{j=2 l}^{\tilde{N} l} \mathcal{O}\left(\hbar^{j}\right)\left(\sum_{k}^{L(\tilde{N}, l)} d_{k} \Phi_{G(k)}\right)
$$

where $d_{k}>0$ are constants, $L(\tilde{N}, l)>0$ is a large (but computable) constant, and $G(k) \in \mathbb{N}^{n}$. Moreover the $L^{2}$ norm of each term of (5.17) applied to our ground state, keeping in mind that $U_{\text {quad }}(t)$ is unitary, is

$$
\left\|\frac{t^{p}}{p !(i \hbar)^{p}} U_{\text {quad }}(t)\left(\tilde{Q}_{\text {quad }}+Q_{\mathrm{nq}}^{(\tilde{N})}\right)^{p} \Phi_{0}\right\| \leq C_{p, \tilde{N}, r}\left(|t|^{p} \hbar^{p}\right)
$$

The constant $C_{p, \tilde{N}}$ grows exponentially in $\tilde{N}$ but since $\tilde{N}$ is independent of $\hbar$ this is not an issue.

We now return to the remainder term $R_{l}^{(N)}(t, \hbar)$ from $(5.17)$. It yields

$$
\left\|\frac{1}{(i \hbar)^{l+1}} \int_{0}^{t} \frac{t_{l}^{l}}{l !} U\left(t-t_{l+1}\right) U_{\text {quad }}\left(t_{l+1}\right)\left(\tilde{Q}_{\text {quad }}+Q_{\mathrm{nq}}^{(\tilde{N})}\right)^{l+1} \Phi_{0} d t_{l+1}\right\| \leq C_{l, \tilde{N}, r}(|t| \hbar)^{l+1} .
$$

Using these estimates, we group the terms (5.17) in increasing powers of $\hbar$. Although the notation is tedious, it is useful to write the general expression of the $p$-th term in the sum (5.17) (removing the factor $\left.\frac{t^{p}}{p !(i \hbar)^{p}}\right)$ :

$$
\mathcal{O}\left(\hbar^{2 p}\right) \sum_{j_{1}, \ldots, j_{p}}^{N, \ldots, N} \sum_{\left|\alpha^{1}\right|=\left|\beta^{1}\right|=j_{1}, \ldots,\left|\alpha^{p}\right|=\left|\beta^{p}\right|=j_{p}} q_{i_{1}}^{\alpha^{1}, \beta^{1}}(\hbar) \ldots q_{i_{p}}^{\alpha^{p}, \beta^{p}}(\hbar) \mathrm{Op}_{\hbar}^{\mathrm{w}}\left(x^{\alpha^{1}} \xi^{\beta^{1}}\right) \ldots \mathrm{Op}_{\hbar}^{\mathrm{w}}\left(x^{\alpha^{p}} \xi^{\beta^{p}}\right) \Phi_{0}
$$

where $\mathcal{O}\left(\hbar^{2 p}\right)$ denotes a function which is bounded above by a positive constant times $\hbar^{2 p}$. Beyond the principal term $\mathcal{D}_{t \vec{\lambda}} \Phi_{0}$, the Dyson expansion then takes the form

$$
\mathcal{D}_{t \rightarrow \lambda}\left[c_{1}(t, h)\left(\sum_{k_{1}}^{L(1, N)} d_{k_{1}} \Phi_{G\left(k_{1}\right)}\right)+c_{2}(t, h)\left(\sum_{k_{2}}^{L(2, N)} d_{k_{2}} \Phi_{G\left(k_{2}\right)}\right)+\cdots+c_{l}(t, h)\left(\sum_{k_{l}}^{L(l, N)} d_{k_{l}} \Phi_{G\left(k_{l}\right)}\right)\right]
$$

where $c_{p}(t, h)=\mathcal{O}\left(|t|^{l} \hbar^{p}\right)$.

5.2. Microlocal support of the evolved state. Fix a small $\varepsilon_{2} \in(0,1)$, For $\hbar \in(0,1 / 2]$ we define the Ehrenfest time

$$
T_{\varepsilon_{2}} \stackrel{\text { def }}{=} \frac{\left(1-\varepsilon_{2}\right)|\log \hbar|}{2 \tilde{\lambda_{1}}},
$$

where $\tilde{\lambda_{1}}$ is the maximal expansion rate amongst the individual rates $\left\{\lambda_{i}\right\}$; in our specific case, $\tilde{\lambda}_{1}=2$. 
Proposition 5.32. Let $\tilde{M}>0$ be an exponent. Let $\Phi_{t}^{(N)}=e^{-i Q^{(\tilde{N})} t / \hbar} \Phi_{0}$, with its expansion given in Proposition 5.13, and set $l=\tilde{M}$. Take $\Theta \in C^{\infty}\left(T^{*}\left(\mathbb{R}^{r}\right)\right)$ with $\Theta \equiv 1$ in a neighbourhood of $(0 ; 0)$, and denote its rescaling by $\Theta_{\alpha}(x, \xi) \stackrel{\text { def }}{=} \Theta(x / \alpha, \xi / \alpha)$. Then, for a normal form degree $\tilde{N} \in \mathbb{N}$ given in Proposition 4.14, and $\varepsilon_{2}>0$, there exists $C_{\tilde{M}, \tilde{N}, l}>0$ such that

$$
\left\|\left[\mathrm{Op}_{\hbar}\left(\Theta_{\hbar^{\varepsilon_{2} / 3}}\right)-I\right] \Phi_{t}^{(N)}\right\|_{L^{2}} \leq C_{\tilde{M}, \tilde{N}, l, r} \hbar^{\tilde{M}}, \quad \hbar \in(0,1 / 2],
$$

uniformly for times $t \in\left[-T_{\varepsilon_{2}}, T_{\varepsilon_{2}}\right]$.

Proof. This is a variant of [15, Prop. 4.22]. We begin with cutoffs given by a product, choosing $\theta_{i} \in C_{\mathrm{c}}^{\infty}\left([-2,2]^{2}\right)$ for each $i=1, \ldots, r$ such that $\theta_{i}=1$ in $[-1,1]^{2}$, and scaling them as $\theta_{i, \alpha}(x, \xi) \stackrel{\text { def }}{=} \theta_{i}(x / \alpha, \xi / \alpha)$. It was shown in [15] that for any index $m$ there exists $C_{m}>0$ in a bounded range such that

$$
\left\|\left[\mathrm{Op}_{\hbar}^{\mathrm{w}}\left(\theta_{i, \alpha}\right)-I\right] \mathcal{D}_{t \lambda_{i}} \varphi_{m, i}\right\|_{L^{2}} \leq C_{m} \hbar^{\tilde{M}}
$$

uniformly for $|t| \leq T_{\varepsilon_{2}}$ and width $\alpha \geq \hbar^{\varepsilon_{2} / 3}$.

We propagate the product and use Proposition 5.13 along with the fact that each expression $\sum_{k_{p}}^{L(p, \tilde{N})} d_{k_{p}} \Phi_{G\left(k_{p}\right)}$ is a sum of terms each of which is a product of scaled Gaussians $\varphi_{0}$ in disjoint variables (each of uniform width $e^{t \tilde{\lambda}_{1}} \hbar^{1 / 2} \leq \hbar^{\varepsilon_{2} / 2}$ by (5.31))) multiplied by polynomial factors. As we have a product structure in our evolved state given by Proposition 5.13, we have the same estimate for the individual terms:

$$
\left\|\left[\mathrm{Op}_{\hbar}\left(\prod_{i=1}^{r} \theta_{i, \alpha}\right)-I\right] \mathcal{D}_{t \vec{\lambda}} \Phi_{G(p)}\right\|_{L^{2}} \leq C_{\tilde{M}, \tilde{N}, r} \hbar^{M}
$$

uniformly for $|t| \leq T_{\varepsilon_{2}}$, multi-indices $G(p)$ in a bounded range, and the same width $\alpha \geq \hbar^{\varepsilon_{2} / 3}$ in all variables where $C_{N, p}>0$. As the number of terms in the sum (5.14) depends only on $l$ and not on $\hbar$, this estimate holds for $\Phi_{t}^{(N)}$ as well but now with a constant $C_{l, \tilde{N}, r}^{\prime}>0$. Since $R_{l}^{(N)}(t, \hbar)=\mathcal{O}\left(\hbar^{l+1-\delta}\right)$ for some $\delta>0$ arising from the fact that $t \in\left[-T_{\varepsilon_{2}}, T_{\varepsilon_{2}}\right]$, we set $l=M$ as the number of iterations in the Dyson expansion which in turn give us the following estimate for the full state $\Phi_{t}^{(N)}$ :

$$
\left\|\left[\mathrm{Op}_{\hbar}\left(\prod_{i=1}^{r} \theta_{i, \alpha}\right)-I\right] \Phi_{t}^{(N)}\right\|_{L^{2}} \leq C_{\tilde{M}, \tilde{N}, r}^{\prime} \hbar^{M} .
$$

Now, consider a non-product form cutoff $\Theta_{\alpha} \in C_{\mathrm{c}}^{\infty}\left(\mathbb{R}^{2 r}\right)$ equal to 1 on the support of $\prod_{i=1}^{r} \theta_{i}$. Then

$$
\Theta_{\alpha}-1=\left(1-\Theta_{\alpha}\right)\left(\prod_{i=1}^{r} \theta_{i, \alpha}-1\right) .
$$

Taking $\alpha \geq \hbar^{\varepsilon_{2} / 3}, \varepsilon_{2} / 3<1 / 2$ so that the semiclassical symbol class $S_{\varepsilon_{2} / 3}^{0}$ (see (3.5)) continues to have expansions in terms of increasing powers of $\hbar$, and using the support properties of $\Theta_{\alpha}$ yields pseudodifferential cutoffs of the form

$$
\mathrm{Op}_{\hbar}^{\mathrm{w}}\left(\Theta_{\alpha}-1\right)=\mathrm{Op}_{\hbar}^{\mathrm{w}}\left(1-\Theta_{\alpha}\right) \mathrm{Op}_{\hbar}^{\mathrm{w}}\left(\prod_{i=1}^{r} \theta_{i, \alpha}-1\right)+\mathcal{O}_{L^{2}}\left(\hbar^{\infty}\right)
$$


Our proof is complete after applying this operator to $\Phi_{t}^{(N)}$ and using (5.35).

\section{A LOG-SCAle QuASimode FOR The BirkHofF NORMAL FORM}

A straightforward calculuation gives $\left\|Q^{(N)}(\hbar) \Phi_{0}\right\|=\left(\prod_{i=1}^{r} \sqrt{2} \lambda_{i}\right) \hbar+\mathcal{O}_{N}\left(\hbar^{2}\right)$ after recalling that $\Phi_{0}$ is the standard Gaussian of equation (5.2). Therefore applying the unitary Fourier integral operators of Proposition 4.14 to the state constructed so far produces a quasimode $\boldsymbol{\Psi}_{\hbar}$ for equation (4.1) with a spectral width which is too large.

To get a narrower quasimode we use the usual time-averaging procedure originally due to Vergini-Schneider [29] and also employed in the work [15].

Let $T>0$ be an averaging time which will be chosen later, fix a weight function $\chi \in$ $C_{\mathrm{c}}^{\infty}((-1,1),[0,1])$ and its rescaled version $\chi_{T}(t) \stackrel{\text { def }}{=} \chi(t / T)$. Our transverse quasimode will be

$$
\tilde{\Phi}_{\chi_{T}, \hbar}^{(\tilde{N})} \stackrel{\text { def }}{=} \int_{\mathbb{R}} \chi_{T}(t) e^{i t\left(E_{\hbar}-1\right) / \hbar} \Phi_{t}^{(\tilde{N})} d t
$$

where $E_{\hbar}=1+f(\hbar)$. Note that this state is not yet normalized. In order to compute its spectral width, we will first need to compute its $L^{2}$ norm.

LEMma 6.2. Let $C>0$ be a constant which will be chosen later. For the semiclassically large averaging time $1 \leq T=T_{\hbar} \leq C|\log \hbar|$ the square norm of our state $\tilde{\Phi}_{\chi_{T}, \hbar}^{(\tilde{N})}$ satisfies

$$
\left\|\tilde{\Phi}_{\chi_{T}, \hbar}^{(\tilde{N})}\right\|^{2}=T S(\lambda, f(\hbar) / \hbar, r)\|\chi\|_{L^{2}}^{2}\left(1+\mathcal{O}_{\tilde{N}, r}(1 / T)\right),
$$

where $S(\bullet, \bullet, \bullet)$ is a positive (and explicit) function of $\hbar$ (small enough) and $\lambda$, which is the vector of expansion rates transverse to $M$ as seen in Proposition 4.14 .

Proof. Although we obtained an explicit expression for the Dyson series (5.14), we prefer here the slightly less explicit operator equation (5.17) in order to reduce our calculations to those in the proof of [15, Lem. 5.4].

We begin with the representation of the evolved state as

$$
\Phi_{t}^{(\tilde{N})}=\left(U_{\text {quad }}(t)+\sum_{p=1}^{l} \frac{t^{p}}{p !(i \hbar)^{p}} U_{\text {quad }}(t)\left(Q_{\mathrm{nq}}^{(\tilde{N})}\right)^{p}+R_{l}\right) \Phi_{0}
$$

for some $l \in \mathbb{N}$ to be determined later. The norm squared of the averaged quasimode is then

$$
\left\|\tilde{\Phi}_{\chi_{T}, E_{\hbar}}^{(\tilde{N})}\right\|^{2}=\iint e^{-i\left(t^{\prime}-t\right) f(\hbar) / \hbar}\left\langle\Phi_{t^{\prime}}^{(\tilde{N})}, \Phi_{t}^{(\tilde{N})}\right\rangle \chi_{T}\left(t^{\prime}\right) \chi_{T}(t) d t d t^{\prime} .
$$

The key is then approximating the overlaps $\left\langle\mathcal{D}_{t \vec{\lambda}}\left(Q_{\mathrm{nq}}^{(\tilde{N})}\right)^{p} \Phi_{0}, \mathcal{D}_{t^{\prime} \vec{\lambda}}\left(Q_{\mathrm{nq}}^{(\tilde{N})}\right)^{p^{\prime}} \Phi_{0}\right\rangle$ $=\left\langle\left(Q_{\mathrm{nq}}^{(\tilde{N})}\right)^{p} \Phi_{0}, \mathcal{D}_{\left(t^{\prime}-t\right) \vec{\lambda}}\left(Q_{\mathrm{nq}}^{(\tilde{N})}\right)^{p^{\prime}} \Phi_{0}\right\rangle$.

Using again the resonance condition on the Weyl symbols we have that $\left(Q_{\mathrm{nq}}^{(\tilde{N})}\right)^{p}$ is a power of a symmetric operator and that $\left(Q_{\mathrm{nq}}^{(\tilde{N})}\right)^{p}$ commutes with $\mathcal{D}_{t \vec{\lambda}}$. From these

$$
\left\langle\left(Q_{\mathrm{nq}}^{(\tilde{N})}\right)^{p} \Phi_{0}, \mathcal{D}_{\left(t^{\prime}-t\right) \vec{\lambda}}\left(Q_{n q}^{(\tilde{N})}\right)^{p^{\prime}} \Phi_{0}\right\rangle=\left\langle\left(Q_{\mathrm{nq}}^{(\tilde{N})}\right)^{p+p^{\prime}} \Phi_{0}, \mathcal{D}_{\left(t^{\prime}-t\right) \vec{\lambda}} \Phi_{0}\right\rangle
$$


Since for our Euclidean quantization $\mathrm{Op}_{\hbar}^{\mathrm{w}}\left(x^{\alpha} \xi^{\beta}\right)=\prod_{i=1}^{r} \mathrm{Op}_{\hbar}^{\mathrm{w}}\left(x_{i}^{\alpha_{i}} \xi_{i}^{\beta_{i}}\right)$, the operator $\left(Q_{\mathrm{nq}}^{(\tilde{N})}\right)^{p}$ maintains a product-type form into sums of differential operators in disjoint variables, it suffices to estimate products of the form

$$
\prod_{i=1}^{r}\left\langle\sum_{m_{i} \geq 0}^{K\left(\tilde{N}, p, p^{\prime}\right)} c_{m_{i}} \varphi_{m_{i}}, \mathcal{D}_{\left(t^{\prime}-t\right) \lambda_{i}} \varphi_{0}\right\rangle_{\mathbb{R}_{x_{i}}}
$$

where $K\left(\tilde{N}, p, p^{\prime}\right)>0$.

A straightforward calculation shows

$$
\left\langle\varphi_{0}, \mathcal{D}_{\left(t^{\prime}-t\right) \lambda_{i}} \varphi_{0}\right\rangle_{\mathbb{R}_{x_{i}}}=\frac{1}{\sqrt{\cosh \lambda_{i}\left(t^{\prime}-t\right)}} .
$$

A similar identity for excited states is developed in [15, Sec. 5.1] and gives for each $m \in \mathbb{N}$ a constant $C_{m}>0$ such that

$$
\left|\frac{\left\langle\varphi_{m}, \mathcal{D}_{\left(t^{\prime}-t\right) \lambda_{i}} \varphi_{0}\right\rangle_{\mathbb{R}_{x_{i}}}}{\left\langle\varphi_{0}, \mathcal{D}_{\left(t^{\prime}-t\right) \lambda_{i}} \varphi_{0}\right\rangle_{\mathbb{R}_{x_{i}}}}\right| \leq C_{m} \text { uniformly in } t, t^{\prime} \in \mathbb{R}
$$

(in fact $C_{m}=0$ for odd $m$ since in that case we are integrating an odd function against an even function).

Returning to equation (6.4), the term arising from the quadratic operator $U_{\text {quad }}(t)$ which corresponds to the case $m=0$ takes the form

$$
\begin{aligned}
& I_{0}=\int e^{-i\left(t^{\prime}-t\right) f(\hbar) / \hbar}\left(\prod_{i=1}^{r}\left\langle\varphi_{0}, \mathcal{D}_{\left(t-t^{\prime}\right) q^{1}} \varphi_{0}\right\rangle_{\mathbb{R}_{x_{i}}}\right) \chi_{T}\left(t^{\prime}\right) \chi_{T}(t) d t d t^{\prime} \\
& =\int e^{-i\left(t^{\prime}-t\right) f(\hbar) / \hbar}\left(\prod_{i=1}^{r} \frac{1}{\sqrt{\cosh \left(q_{1, i}(\hbar)\left(t^{\prime}-t\right)\right)}}\right) \chi_{T}\left(t^{\prime}\right) \chi_{T}(t) d t d t^{\prime} .
\end{aligned}
$$

A similar expression was evaluated in [15, Sec. 5.1], giving

$$
I_{0}(\hbar)=T S(\vec{\lambda}, f(\hbar) / \hbar, r)\|\chi\|_{L^{2}}^{2}\left(1+\mathcal{O}_{r}(1 / T)\right)
$$

It is also shown there that the correction terms arising from $m>0$ (and the remainder $R_{l}$ ) are bounded above by $\tilde{C}_{m} \hbar T^{l} I_{0}(0)$ when $\hbar$ is small enough. We have taken $T \sim|\log \hbar|$ so the correction terms are $\mathcal{O}\left(\hbar^{\delta}\right)$ for some $\delta>0$ and are therefore lower order than the constant appearing in (6.10).

For this we need to bound $I_{0}(\hbar)$, that is $S(\vec{\lambda}, f(\hbar) / \hbar, r)$, above and below. We would like to do this uniformly for $\hbar$ small enough, as this uniformity feeds into the argument for Corollary 1.10. As $f(\hbar) / \hbar=\mathcal{O}(1)$ and $S(\vec{\lambda}, f(\hbar) / \hbar, r)$ is the 1-dimensional Fourier transform of a non-zero Schwartz function (for all $\hbar$ ), the positivity of $\left\|\tilde{\Phi}_{\chi_{T}, E_{\hbar}}^{(\tilde{N})}\right\|^{2}$ establishes that of $S$.

We can now define our penultimate normalized state

$$
\Psi_{\chi_{T}, \hbar}^{(N)} \stackrel{\text { def }}{=} \frac{\tilde{\Phi}_{\chi_{T}, \hbar}^{(N)}}{\left\|\tilde{\Phi}_{\chi_{T}, h}^{(N)}\right\|}
$$


Corollary 6.12. Given $\varepsilon_{2}>0$ we may choose an averaging time $T=T_{\varepsilon_{2}} \leq C\left(\varepsilon_{2}\right)|\log h|$ where $C\left(\varepsilon_{2}\right)>0$ such that the normalized state $\Psi_{\chi_{T}, h}^{(\tilde{N})}$ is localized in the $\hbar^{\varepsilon_{2} / 3}$ neighbourhood of $(0 ; 0)$. That is, for any $\Theta \in C_{\mathrm{c}}^{\infty}(\mathbb{R})$ with $\Theta \equiv 1$ in a fixed neighbourhood of $(0 ; 0)$, we have the estimate

$$
\left\|\left[\mathrm{Op}_{\hbar}\left(\Theta_{\hbar^{\varepsilon_{2} / 3}}\right)-I\right] \Psi_{\chi_{T}, \hbar}^{(\tilde{N})}\right\|_{L^{2}}=\mathcal{O}_{\tilde{N}}\left(\hbar^{\infty}\right) .
$$

Proof. This is immediate from Proposition 5.32 whilst keeping in mind that $T_{\varepsilon_{2}}$ goes to the Ehrenfest time (5.31).

Proposition 6.14. For $T=T_{\varepsilon_{2}}$, the spectral width of the state $\Psi_{\chi_{T}, h}^{(\tilde{N})}$ at energy 0 is

$$
\left\|\left(Q^{(\tilde{N})}(\hbar)-f(\hbar)\right) \Psi_{\chi_{T}, \hbar}^{(\tilde{N})}\right\|^{2}=\frac{\hbar^{2}}{T_{\varepsilon_{2}}^{2}} \frac{\left\|\chi^{\prime}\right\|_{L^{2}}^{2}}{\|\chi\|_{L^{2}}^{2}}\left(1+\mathcal{O}_{\tilde{N}, r}(1 /|\log \hbar|)\right) .
$$

Hence, $\left\{\Psi_{\chi_{T}, h}\right\}_{\hbar}$ yields a semiclassical measure which is invariant under the classical dynamics generated by $Q^{(\tilde{N})}(h)$ and equal to $\delta_{0}(x) \in \mathcal{D}^{\prime}\left(\mathbb{R}^{r}\right)$.

Proof. Recalling that $\Psi_{\chi_{T}, \hbar}$ is defined by (6.1), we perform the following calculation:

$$
\begin{aligned}
\left(Q^{(\tilde{N})}(h)-f(\hbar)\right) \Psi_{\chi_{T}, h} & =\int_{\mathbb{R}} \chi_{T}(t)\left(Q^{(\tilde{N})}(h)-f(\hbar)\right) e^{-i t\left(Q^{(\tilde{N})}(h)-f(\hbar)\right) / \hbar} \Phi_{0} d t \\
& =\int_{\mathbb{R}} \chi_{T}(t) i \hbar \partial_{t}\left(e^{-i t\left(Q^{(\tilde{N})}(h)-f(\hbar)\right) / \hbar}\right) \Phi_{0} d t \\
& =-i \hbar \int_{\mathbb{R}}\left(\partial_{t} \chi_{T}(t)\right) e^{-i t\left(Q^{(\tilde{N})}(h)-f(\hbar)\right) / \hbar} \Phi_{0} d t
\end{aligned}
$$

The norm of the last integral was essentially computed in Lemma 6.2 except our cutoff in time is now $-i \hbar \chi^{\prime}(t / T) / T$. A division by the asymptotic formula in the same Lemma for $\left\|\Psi_{\chi_{T}, \hbar}\right\|$ finishes the first statement of our proposition.

The second statement follows from our quasimode having spectral width which is clearly $o(\hbar)$ and therefore yielding an invariant semiclassical measure under $\exp \left(t H_{q^{(\tilde{N})}}\right)[32]$. The microlocal support statement from Corollary 6.12 tells us that this can only be $\delta_{0}$.

The spectral width of the normalized mode $\tilde{\Psi}_{\chi_{T}, h}^{(\tilde{N})}$ then takes the form

$$
F(\hbar)=\frac{\hbar}{T_{\varepsilon_{2}}} \frac{\left\|\chi^{\prime}\right\|_{L^{2}}}{\|\chi\|_{L^{2}}}\left(1+\mathcal{O}_{\tilde{N}, r}(1 /|\log \hbar|)\right)
$$

We note that the ratio $\frac{\left\|\chi^{\prime}\right\|_{L^{2}}}{\|\chi\|_{L^{2}}}$ is essentially a universal constant. Since $[15$, Lem. 5.16] determined the infimum of these ratios to be $\pi / 2$, we make the slight improvement of choosing $\chi$ for which the ratio is $\left(1+\varepsilon_{2}\right) \pi / 2$. The resulting spectral width is therefore

$$
\begin{aligned}
F(\hbar) & =\pi \tilde{\lambda}_{1} \frac{1+\varepsilon_{2}}{1-\varepsilon_{2}} \frac{\hbar}{|\log h|}+\mathcal{O}_{\tilde{N}, r}\left(\frac{h}{|\log h|^{2}}\right) \\
& \leq \pi \tilde{\lambda}_{1}\left(1+3 \varepsilon_{2}\right) \frac{\hbar}{|\log h|}+\mathcal{O}_{\tilde{N}, r}\left(\frac{h}{|\log h|^{2}}\right)
\end{aligned}
$$

where $\tilde{\lambda}_{1}=2$. 


\section{A log-SCAle quasimode on $N$ And the Proof of Theorem 1.9}

We now set $C=\pi \tilde{\lambda}_{1}\left(1+3 \varepsilon_{2}\right)$. Having constructed quasimodes of spectral width $\frac{C \hbar}{|\log \hbar|}$ for our transverse dynamics in the Birkhoff Normal Form coordinates, we would like to transport them into our collar neighborhood $N_{\varepsilon_{1}}(M)$ and plug them into our ansatz. The necessary ingredient is cutting off $\Psi_{\chi_{T}, \hbar}$ in space.

We revert to our local Fermi-normal coordinate system $\left(\eta, w^{\prime}, x\right)$ in the collar neighbourhood $N_{\varepsilon_{1}}(M)$ determined in Section 2 where $\left(\eta, w^{\prime}\right)$ are local coordinates on $M$ and $x$ is our $r$-dimensional transverse variable. Consider the energy $E_{\hbar}=1+f(\hbar)$.

By Lemma 4.5, it suffices to analyze the behavior of the result of transporting the quasimode of the previous section to a quasimode for $K_{x}(\hbar)$ in the $x$ variable, which we proceed to do.

Let $\Upsilon \in C_{\mathrm{c}}^{\infty}\left(\mathbb{R}^{r}\right)$ be supported on $\left[-\varepsilon_{1} / 2, \varepsilon_{1} / 2\right]^{r}$ and equal to 1 on $\left[-\varepsilon_{1} / 3, \varepsilon_{1} / 3\right]^{r}$. Choose $\varepsilon_{2}>0$ which is related to our semiclassical averaging time. We set $\psi_{\hbar}=\Upsilon(x) U_{\tilde{N}}\left(\Psi_{\chi_{T}, h}^{(\tilde{N})}\right)$ and set

$$
\boldsymbol{\Psi}_{\hbar}=\varphi_{\hbar}\left(\eta, w^{\prime}\right) \psi_{\hbar}(x) .
$$

In the definition of $\psi_{\hbar}, U_{\tilde{N}}(\hbar)$ is the microlocally unitary FIO discussed in Proposition 4.14 and $\Psi_{\chi_{T}, \hbar}^{(\tilde{N})}$ is as in equation (6.1). Then for $\hbar \leq \hbar_{0}\left(\varepsilon_{1}\right), \psi_{\hbar}$ will be supported in the range of the transverse variable $u$ permitted in the collar neighbourhood making our function $\boldsymbol{\Psi}_{\hbar}$ well-defined. We need to verify that the cutoff $\Upsilon$ does not affect the norm, spectral behavior, and concentration properties.

For the norm, Corollary 6.12 and properties of Fourier integral operators associated to canonical transformations (particularly those appearing Proposition 4.14) tell us that for $\hbar \leq \hbar_{0}\left(\varepsilon_{1}\right)$ small enough,

$$
\left\|\Upsilon U_{\tilde{N}}\left(\Psi_{\chi_{T}, \hbar}^{(\tilde{N})}\right)\right\|_{L^{2}\left(\mathbb{R}^{r},\left(1+|x|^{2}\right)^{\frac{m-1}{2}} d x\right)}=\left\|\Psi_{\chi_{T}, \hbar}^{(\tilde{N})}\right\|+\mathcal{O}_{\tilde{N}}\left(\hbar^{\infty}\right)=1+\mathcal{O}_{\tilde{N}}\left(\hbar^{\infty}\right) .
$$

Note that the statement of Corollary 6.12 is exactly that $\left\{\psi_{\hbar}\right\}_{\hbar}$ concentrate at 0 in the Birhkoff normal form coordinates. Proposition 4.14 demonstrates that this concentration carries to concentration near $x=0$ for $\left\{U_{\tilde{N}}\left(\tilde{\Psi}_{\chi_{T}, \hbar}^{(\tilde{N})}\right)\right\}_{\hbar}$ and multiplying by the cutoff $\Upsilon$ does not affect that for $\hbar \leq \hbar_{0}\left(\varepsilon_{1}\right)$.

For the spectral width, we need to verify that applying the FIO $U_{N}$ and multiplication by $\Upsilon$ have negligible effect. Concerning $U_{\tilde{N}}$, we have

$$
\begin{gathered}
\left\|K_{x}(\hbar)\left(\Upsilon U_{\tilde{N}}\left(\Psi_{\chi_{T}, \hbar}^{(\tilde{N})}\right)\right)\right\|_{L^{2}\left(\mathbb{R}^{r},\left(1+|x|^{2}\right)^{\frac{m-1}{2}} d x\right)}= \\
\left\|U_{\tilde{N}}\left(\left(Q^{(\tilde{N})}(\hbar)+R_{\tilde{N}+1}(\hbar)\right) \Psi_{\chi_{T}, \hbar}^{(\tilde{N})}\right)\right\|+\mathcal{O}_{\tilde{N}, r}\left(\hbar^{\infty}\right)
\end{gathered}
$$

for $\hbar$ small enough. Proposition 6.14 along with our optimized cutoffs $\chi$ then give:

$$
\left\|U_{\tilde{N}}\left(Q^{(\tilde{N})}(\hbar) \Psi_{\chi_{T}, h}^{(\tilde{N})}\right)\right\|=\pi \tilde{\lambda}_{1}\left(1+3 \varepsilon_{2}\right) \frac{\hbar}{|\log \hbar|}+\mathcal{O}_{\tilde{N}, r}\left(\frac{\hbar}{|\log \hbar|^{2}}\right) .
$$

The contribution from the remainder $R^{(\tilde{N}+1)}(\hbar)$ is small: a second application of Corollary 6.12 tells us that $R^{(\tilde{N}+1)}(\hbar) \Psi_{\chi_{T}, \hbar}^{(\tilde{N})}=R^{(\tilde{N}+1)}(\hbar) \Theta_{\hbar_{2} / 3}(\hbar) \tilde{\Psi}_{\chi_{T}, \hbar}^{(\tilde{N})}+\mathcal{O}_{\tilde{N}, r}\left(\hbar^{\infty}\right)$. The composition 
calculus for Weyl quantizations gives $R^{(\tilde{N}+1)}(\hbar) \Theta_{\hbar^{\varepsilon_{2} / 3}}(\hbar)=\mathrm{Op}_{\hbar}^{\mathrm{w}}\left(r_{\tilde{N}+1} \sharp \Theta_{\hbar^{\varepsilon_{2} / 3}}\right)$. The Weyl symbol of this Moyal product satisfies the bound

$$
\mathcal{O}_{r}\left(\hbar^{(\tilde{N}+1) \varepsilon_{2} / 3}\right)
$$

after using the symbol estimates in Proposition 4.14. Hence, letting $(\tilde{N}+1) \varepsilon_{2} / 3>1$ and recalling that $l \geq 1$ from the Dyson expansion estimate (5.14) guarantees that our various remainder estimates are $o\left(\hbar /|\log \hbar|^{2}\right)$.

Finally, multiplication by $\Upsilon$ has no effect since the symbol of $\Upsilon$ is the constant 1 near 0 and we have chosen $\hbar \leq \hbar_{0}(\varepsilon)$ so that the quasimode is already concentrated near 0 before the multiplication. This completes the proof of statement (1) of Theorem 1.9.

Remark 7.6. We record here the order in which the various parameters in the construction have been chosen

- Fix a codimension $r<n=\operatorname{dim} N$.

- Given $M \subset N$ of codimension $r$, choose $\varepsilon_{1}>0$ such that the collar neighborhood $N_{\varepsilon_{1}}(M)$ behaves as expected, including in supporting a convenient coordinate system.

- Choose $\varepsilon_{2}>0$, giving the averaging time $T_{\varepsilon_{2}}$.

- Choose $\tilde{N}>0$ such that $\tilde{N}+1>3 / \varepsilon_{2}$.

- Choose any $l>1$.

- Determine $\hbar_{0}$ depending on all the previous choices such that if $0<\hbar<\hbar_{0}$ then the final state $\boldsymbol{\Psi}_{\hbar}$ is microlocalized within the collar neighbourhood $N_{\varepsilon_{1}}(M)$.

The following lemma is a slight restatement of that in Section 6 of [15] and proves statement (2) of our Theorem 1.9. We note that its proof is independent of whether the support of the invariant semiclassical measure $\mu_{s c}$ associated to $\left\{\boldsymbol{\Psi}_{\hbar}\right\}_{\hbar}$ is smooth or not. Therefore, we leave it to the interested reader for details.

LEMmA 7.7 (Partial localization on invariant subsets). Let $\varepsilon_{2}, \varepsilon_{3}>0$ be given. Let $E_{0}>0$ be a regular energy level of $-h^{2} \Delta_{N}$, and $\mu_{F}$ be any invariant semiclassical measure on $S_{E_{0}} N$ with support $F$ a closed set. Let $C_{\text {width }}=\pi \tilde{\lambda}_{1}\left(1+3 \varepsilon_{2}\right)$ be the $\hbar$-independent factor in the spectral width of the fully localized mode $\boldsymbol{\Psi}_{h}$ defined in (7.1). Then there exists quasimodes $\tilde{\boldsymbol{\Psi}}_{h}$ of spectral width $\varepsilon_{3} \frac{h}{|\log h|}$ and central energies $E_{\hbar}=E_{0}+\mathcal{O}\left(\frac{\hbar}{|\log \hbar|}\right)$ whose semiclassical measure on $S_{E_{0}} N$ contains an atom $w_{F} \cdot \mu_{F}$ in its ergodic decomposition where

$$
w_{F} \geq \frac{\varepsilon_{3}}{C_{\text {width }}} \frac{2}{3 \sqrt{3}}+\mathcal{O}\left(\left(\frac{\varepsilon_{3}}{C_{\text {width }}}\right)^{2}\right) .
$$

The proof of statement (3) of Theorem 1.9 then follows by replacing the tangential eigenfunction $\varphi_{h}(7.1)$ with a log-scale quasimode and applying Lemma 4.5.

\section{REFERENCES}

1. Ralph Abraham and Jerrold E. Marsden, Foundations of mechanics, Benjamin/Cummings Publishing Co., Inc., Advanced Book Program, Reading, Mass., 1978, Second edition, revised and enlarged, With the assistance of Tudor Ratiu and Richard Cushman. MR 515141

2. Nalini Anantharaman, Entropy and the localization of eigenfunctions, Ann. of Math. (2) 168 (2008), no. 2, 435-475. MR 2434883 
3. __ A hyperbolic dispersion estimate, with applications to the linear Schrödinger equation, Proceedings of the International Congress of Mathematicians. Volume III (New Delhi), Hindustan Book Agency, 2010, pp. 1839-1861. MR 2827869

4. Nalini Anantharaman, Herbert Koch, and Stéphane Nonnenmacher, Entropy of eigenfunctions, New Trends in Mathematical Physics, Selected contributions of the XVth International Congress on Mathematical Physics (Vladas Sidoraviius, ed.), Springer, 2006, arXiv:math-ph/0704.1564, pp. 1-22.

5. Nalini Anantharaman and Stéphane Nonnenmacher, Entropy of semiclassical measures of the walshquantized bakers map, Ann. Henri Poincaré (2007), no. 8, 37-74.

6. Nalini Anantharaman and Stéphane Nonnenmacher, Half-delocalization of eigenfunctions for the Laplacian on an Anosov manifold, Ann. Inst. Fourier (Grenoble) 57 (2007), no. 7, 2465-2523, Festival Yves Colin de Verdière. MR 2394549 (2009m:81076)

7. Nalini Anantharaman and Lior Silberman, A Haar component for quantum limits on locally symmetric spaces, Israel J. Math. 195 (2013), no. 1, 393-447. MR 3101255

8. Shimon Brooks, Logarithmic-state quasimodes that do not equidistribute, Int. Math. Res. Not. IMRN (2015), no. 22, 11934-11960. MR 3456709

9. Shimon Brooks and Elon Lindenstrauss, Joint quasimodes, positive entropy, and quantum unique ergodicity, Invent. Math. 198 (2014), no. 1, 219-259. MR 3260861

10. Yves Colin de Verdière, Ergodicité et fonctions propres du laplacien, Comm. Math. Phys. 102 (1985), no. 3, 497-502. MR 87d:58145

11. Yves Colin de Verdière and Bernard Parisse, Équilibre instable en régime semi-classique. I. Concentration microlocale, Comm. Partial Differential Equations 19 (1994), no. 9-10, 1535-1563. MR MR1294470 (96b:58112)

12. __ Équilibre instable en régime semi-classique. I. Concentration microlocale, Comm. Partial Differential Equations 19 (1994), no. 9-10, 1535-1563. MR 1294470

13. M. Combescure and D. Robert, Semiclassical spreading of quantum wave packets and applications near unstable fixed points of the classical flow, Asymptot. Anal. 14 (1997), no. 4, 377-404. MR 1461126

14. Moesz Dimassi and Johannes Sjostrand, Spectral asymptotics in the semiclassical limit, London Mathematical Society, Lecture Note Series 268, 199, First Edition.

15. Suresh Eswarathasan and Stéphane Nonnenmacher, Strong scarring of logarithmic quasimodes, to appear in Ann. Inst. Fourier, preprint available at arXiv:math.SP/1507.08371, 2015.

16. Frédéric Faure, Stéphane Nonnenmacher, and Stephan De Bièvre, Scarred eigenstates for quantum cat maps of minimal periods, Comm. Math. Phys. 239 (2003), no. 3, 449-492. MR MR2000926 (2005a:81076)

17. Patrick Gerard and Eric Leichtnam, Ergodic properties of eigenfunctions for the dirichlet problem, Duke Math. J. 71 (1993), 559-607.

18. Andrew Hassell, Ergodic billiards that are not quantum unique ergodic, Ann. of Math. (2) 171 (2010), no. 1, 605-619, With an appendix by the author and Luc Hillairet. MR 2630052 (2011k:58050)

19. Eric J. Heller, Bound-state eigenfunctions of classically chaotic Hamiltonian systems: scars of periodic orbits, Phys. Rev. Lett. 53 (1984), no. 16, 1515-1518. MR MR762412 (85k:81055)

20. Lars Hörmander, The analysis of linear partial differential operators. III, Grundlehren der Mathematischen Wissenschaften [Fundamental Principles of Mathematical Sciences], vol. 274, Springer-Verlag, Berlin, 1994, Pseudo-differential operators, Corrected reprint of the 1985 original. MR 95h:35255

21. Alexei Iantchenko, La forme normale de Birkhoff pour un opérateur intégral de Fourier, Asymptot. Anal. 17 (1998), no. 1, 71-92. MR 1632700

22. Vladimir F. Lazutkin, KAM theory and semiclassical approximations to eigenfunctions, Ergebnisse der Mathematik und ihrer Grenzgebiete (3) [Results in Mathematics and Related Areas (3)], vol. 24, Springer-Verlag, Berlin, 1993, With an addendum by A. I. Shnirel'man. MR MR1239173 (94m:58069)

23. Elon Lindenstrauss, Invariant measures and arithmetic quantum unique ergodicity, Ann. of Math. (2) 163 (2006), no. 1, 165-219. MR MR2195133

24. Gabriel Rivière, Entropy of semiclassical measures for nonpositively curved surfaces, Ann. Henri Poincaré 11 (2010), no. 6, 1085-1116. MR 2737492

25. Zeév Rudnick and Peter Sarnak, The behaviour of eigenstates of arithmetic hyperbolic manifolds, Comm. Math. Phys. 161 (1994), no. 1, 195-213. MR 95m:11052 
26. Karl Sigmund, On the space of invariant measures for hyperbolic flows, Amer. J. Math. 94 (1972), 31-37. MR 0302866

27. Johannes Sjöstrand, Resonances associated to a closed hyperbolic trajectory in dimension 2, Asymptot. Anal. 36 (2003), no. 2, 93-113. MR 2021528

28. Alexander I. Šnirel'man, Ergodic properties of eigenfunctions, Uspekhi Mat. Nauk 29 (1974), no. 6(180), 181-182. MR 53 \#6648

29. Eduardo G. Vergini and David Schneider, Asymptotic behaviour of matrix elements between scar functions, J. Phys. A 38 (2005), no. 3, 587-616. MR 2116626

30. Steven Zelditch, Uniform distribution of eigenfunctions on compact hyperbolic surfaces, Duke Math. J. 55 (1987), no. 4, 919-941. MR 89d:58129

31. Steven Zelditch and Maciej Zworski, Ergodicity of eigenfunctions for ergodic billiards, Comm. Math. Phys. 175 (1996), 673-682.

32. Maciej Zworski, Semiclassical analysis, Graduate Studies in Mathematics, vol. 138, American Mathematical Society, Providence, RI, 2012. MR 2952218

School of Mathematics, Cardiff University, Senghennyd Road, Cardiff, Wales, United KINGDOM

E-mail address: eswarathasans@cardiff.ac.uk

University of British Columbia, Department of Mathematics, 1984 Mathematics Road, VANCOUVER, BC V6T 1 Z2

E-mail address: lior@math.ubc.ca 